\title{
How Using Machine Learning Classification as a Variable in Regression Leads to Attenuation Bias and What to Do About It
}

\author{
Han Zhang
}

May 13, 2022

\begin{abstract}
Social scientists have increasingly been applying machine learning algorithms to "big data" to measure theoretical concepts and then using these machinepredicted variables in regression. This article first demonstrates that directly inserting binary predictions (i.e. classification) without regard for prediction error will generally lead to the attenuation bias of slope coefficients or marginal effect estimates. We then propose five estimators with which to obtain consistent estimates of the coefficients. The estimators require validation data; both machine prediction and true values can be used. Monte Carlo simulations are used to demonstrate the effectiveness and robustness of the proposed estimators. We summarize the pattern of usage of machine learning predictions in 12 recent publications in the top social science journals, apply our proposed estimators to four of them, and offer some practical recommendations. We develop an $\mathrm{R}$ package (CCER) to help researchers use the proposed estimators.
\end{abstract}




\section{Introduction}

Machine learning (ML) has increasingly been used by social scientists in recent years (Molina and Garip, 2019; Athey and Imbens, 2019; Grimmer et al., 2021). Scholars have used ML algorithms to forecast social and political events (Cederman and Weidmann, 2017), to improve variable selection (Belloni et al., 2012), and to predict counterfactuals to aid causal inference (Wager and Athey, 2018; Chernozhukov et al., 2018).

This article deals with another common usage of machine learning in the social sciences: the use of machine learning for measurement (Grimmer et al., 2021). Researchers use machine leaning for measurement when they are working with socalled "big data." These big datasets are often in new formats (other than survey/administrative data), such as text, images, videos, and audio clips, and may contain millions of observations. It would take a tremendous effort to manually annotate such a large dataset in order to create measures for theoretically relevant variables. With recent advances of machine learning, researchers can now apply machine learning algorithms to the entire dataset to generate predictions for the concepts they wish to measure. These machine predictions will then be used in subsequent statistical analyses, often as variables in regression models.

We use recent research as a running example. Election fraud in non-democratic regimes bears important consequences, but traditionally researchers have only been able to measure it at the macro-level: whether each election is subject to manipulation or not. Cantú (2019) contributed to the literature by creating microlevel measures of whether each vote-tally was altered or not in a election. He manually labeled some vote-tally images from the 1988 Mexico Presidential Election, trained a Convolutional Neural Network, ${ }^{1}$, and applied the algorithm to the remaining majority of unlabeled 
vote-tally images to predict whether or not each vote tally was altered. Finally, he used the machine-predicted vote tally alternation dummy variable as the dependent variable and regressed it on a battery of independent variables.

Machine learning algorithms unavoidably contain prediction errors. For instance, Cantú (2019)'s algorithm for detecting vote tally alternation, although highly accurate, still predicts around $10 \%$ of the images incorrectly. We conducted a literature search of the top journals in political science, economics, and sociology. We found 12 articles published in the last five years that used ML algorithms to generate key measures of their variables (Section 2). In all 12 articles, researchers inserted the machine-predicted variables into the next-step regressions as if they were error-free. We refer to this practice as naive regression. We also found that binary classifications are much more popular than categorical or continuous predictions, and thus this article focuses on the case of binary ML predictions, also commonly referred to as binary classifications.

Do these ML prediction errors bias the estimated coefficients in naive regression? And what is the direction and size of these biases? To answer these questions, we argue that ML prediction error can be treated as a type of measurement error. Therefore, borrowing from the rich statistics literature on how to address these measurement errors in regression modeling (i.e. the error-in-variables model), we show that when binary ML classifications are used directly in naive regression, regardless of whether the predicted variables are used as independent or dependent variables, attenuation bias in slope coefficients (for simple linear regression) or in marginal effects (for generalized linear models such as logistic regression) occurs in most situations (Section 3). In other words, using binary ML classification shrinks the estimated coefficients or marginal effects in the next-step regression toward zero. The size of the attenuation depends on the accuracy of the prediction: the more prediction errors, the more 
attenuation. Hence, the 12 articles that used ML predictions as variables all suffer from attenuation bias in their effect size estimates. Unlike slope, the naive intercept is also biased, but may be attenuated or inflated.

This article departs from most of the literature on errors-in-variables models in a fundamental way. The methodologies of the measurement error literature were developed with survey or administrative data in mind and do not require scholars to quantify the measurement errors. They have instead taken a different route by deriving complex semi-parametric or nonparametric models that rely on strong but possibly unrealistic assumptions about the distribution of measurement errors (e.g. assuming $F N R=F P R$, or $F N R=0$ ) (Bollinger, 1996; Lewbel, 2000; Frazis and Loewenstein, 2003; Carroll et al., 2006; Schennach, 2016; Meyer and Mittag, 2017). This article requires that the researchers have validation data that contains the true values of the predictions. ${ }^{2}$ Such validation data are automatically available if supervised machine learning is used for measurement: one can hold back a portion of the training data for validation purposes. Such validation data are also easy to create if dictionary methods or pre-trained supervised models are used. We discuss extensively how validation data can be obtained and how to use it to empirically estimate classification error rates (Section 4). In particular, we proposed estimators with which to quantify the variance of error rate estimates, which is an underappreciated point in literature on the errors-in-variables model and is a unique contribution of this article.

The estimated error rates will then be used to obtain consistent estimates of the regression coefficient in the next-step regression (Section 5). Our estimators are agnostic to ML algorithm choices. We discuss five types of estimators that are based on regression on validation data, closed-form analytical correction, maximum likelihood estimation (MLE) procedures, Bootstrap methods, and Bayesian statistics. The Bootstrap and Bayesian estimators in particular are unique contributions of this study, 
which further incorporates the variance of error rates into estimating the variances of next-step regression coefficients. We have developed an open source $\mathrm{R}$ package, CCER (Correcting Classification Error in Regression), to help other researchers use the proposed estimators. ${ }^{3}$

Monte Carlo simulations demonstrate that the proposed estimators can successfully identify the true coefficient, whereas naive regression has serious attenuation biases (Section 6). Moreover, our estimators provide better estimates than naive regression under more challenging situations in which model assumptions are violated and/or explanatory variables are omitted. We also discuss the choice of the size of the validation dataset and the tolerable amount of prediction errors when using our estimators, which are decisions researchers need to make.

We applied our estimators to four published studies that treated ML predictions as true values in next-step regression models (Section 7). After applying our corrections, the effect sizes and standard errors all increased. If the ML predictions in the original studies were relatively accurate, then the results remained statistically significant. If the ML predictions were not accurate enough, then standard errors increased to such an extent that the results were no longer statistically significant.

This article is related to two recent studies which explicitly discuss how to incorporate machine learning prediction errors into estimating downstream quantities of interest to social scientists. First, Hopkins and King (2010) proposed a method to estimate the proportion of documents belonging to one class based on binary ML predictions. We prove that Hopkins and King (2010) is a special case in current research. This article is also inspired by Fong and Tyler (2020), who extended an instrumental variable (IV) estimator first proposed in Lee and Sepanski (1995) to a more complex generalized method of moments (GMM) estimator. We prove that one of our estimators is equivalent to the IV estimator, when the ML classification is used 
as an independent variable and there is no control variable. Fong and Tyler (2020) can handle continuously predicted independent variables. However, Fong and Tyler (2020) only dealt with predicted independent variables; we also provide estimators for predicted dependent variables, a situation that is much more common in empirical research. We also specify the direction and size of the biases caused by using ML classification in regression, whereas Fong and Tyler (2020) only pointed out that the coefficients are biased. Together with these previous studies, this article calls for more robust statistical analyses when future researchers use variables predicted by ML algorithms in their research.

\section{How Social Scientists Use ML Predicted Variables}

We searched for the terms "machine learning," "supervised machine learning," and "prediction" in articles from the past five years in top social science journals: two in political science-American Political Science Review (APSR) and American Journal of Political Science (AJPS); three in economics-American Economic Review (AER), Quarterly Journal of Economics (QJE), and Journal of Political Economy (JPE); and two in sociology-American Sociological Review (ASR) and American Journal of Sociology (AJS). We list the 12 articles we found in Table $1 .^{4}$ Table 1 aims to see what are the common problems in published research in top journals, which provide a road map for developing our methods that address problems in these representative research. We also found six other articles using topic modeling and take topic proportions as variables in next-step regression models (Table A.1). We did not include them in Table 1 because none of the six had validated their results against some external "truth", which is a requirement for applying our estimators (more discussions at the end). 
Every study in Table 1 directly inserted ML predictions into their next-step regression models, although most articles have quantified prediction errors of their algorithms so that we know they are not error-free. As we will demonstrate in next section, treating predictions as they were error-free will lead to attenuation biases in their effect size estimates. Judging from the fact that all the articles were published in top journals, more studies will follow them and will thus make similar errors.

We make some additional observations. Eight articles used binary predictions (No. $1-8$ ), two articles used categorical predictions (No. 9 and 10), and another two articles used continuous predictions (No. 11 and 12) (column "Type"). Hence, this article derives estimators for binary predictions, not only for its mathematical simplicity but also for its popularity in applied research.

Focusing only on the articles that used binary ML classification, seven of the eight articles used ML classification as an outcome variable (column "Predicted"). Hence, we also give relatively more attention to the case of using ML predicted variable as the dependent variable. The preference for using ML classification as the dependent variable may be driven by textbook knowledge on errors-in-variables models: when the mismeasured variable is used as the outcome variable, naive regression will produce consistent estimates of regression coefficients (although standard error estimates will be inflated). ${ }^{5}$ For instance, Crabtree et al. (2020, footnote 9 ) explicitly stated that they used ML predictions as the dependent variable because it "does not affect the unbiasedness of one's parameter estimates...will only makes it harder for us to find statistically significant results."

However, it often goes unnoticed that the above statements only hold if we assume classical measurement error: the measurement error has zero mean and is uncorrelated with the truth. Binary, categorical, and bounded continuous variables always violate this classical measurement error assumption because errors are always nega- 


\begin{tabular}{|c|c|c|c|c|c|c|c|c|c|c|c|c|c|}
\hline \multirow{4}{*}{ 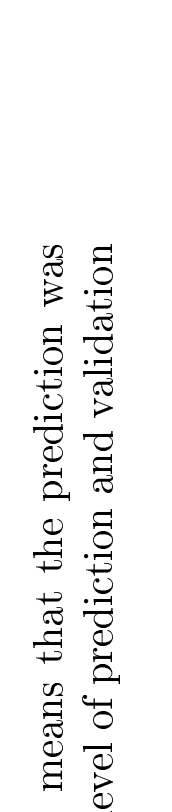 } & 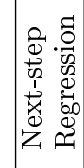 & 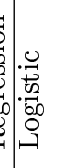 & 芯 & $\stackrel{\widetilde{\varpi}}{\leftrightarrows}$ & 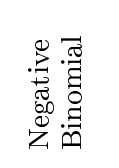 & 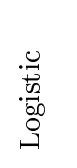 & 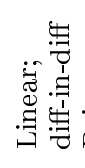 & 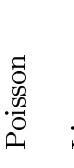 & 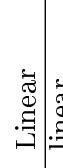 & & 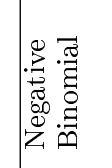 & $\begin{array}{l}\vec{\Phi} \\
\overline{0} \\
\sum^{0} \\
\hat{x}_{0} \\
0\end{array}$ & 离 \\
\hline & 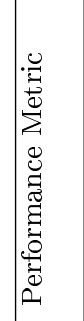 & 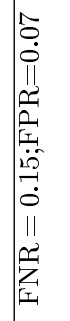 & 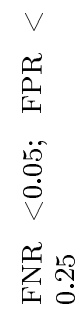 & 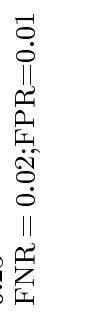 & 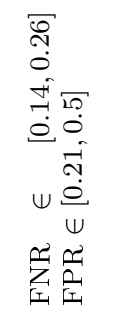 & 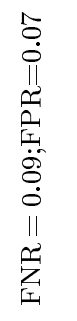 & 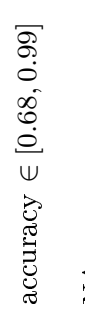 & 茫 & $\vec{z} \mid d$ & & $\vec{z}$ & 岁 & $\overleftrightarrow{z}$ \\
\hline & 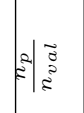 & $\frac{9}{m}$ & 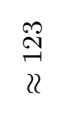 & $\begin{array}{l}\stackrel{\otimes}{\infty} \\
\stackrel{\leftrightarrow}{\wedge}\end{array}$ & $\stackrel{\overbrace{}}{\circ}$ & $\stackrel{\vec{g}}{\rightarrow}$ & $\begin{array}{l}\infty \\
\infty \\
\imath\end{array}$ & 茫; & $\vec{z} \mid d$ & & 岁 & 茫 & $\overleftrightarrow{Z}$ \\
\hline & $\varepsilon^{2}$ & 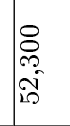 & $\begin{array}{l}\overrightarrow{i n} \\
\stackrel{5}{9} \\
\stackrel{8}{8} \\
\text { c. }\end{array}$ & $\begin{array}{l}\underset{\Delta}{0} \\
\stackrel{\Lambda}{\wedge}\end{array}$ & $\begin{array}{l}\stackrel{9}{9} \\
\stackrel{9}{7} \\
10 \\
-7\end{array}$ & $\begin{array}{l}\mathbb{N} \\
\text { N } \\
18 \\
0\end{array}$ & $\begin{array}{l}m \\
\text { O. } \\
\dot{F}\end{array}$ & $\sum_{\substack{\infty \\
\curvearrowright}}$ & 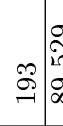 & 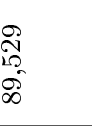 & 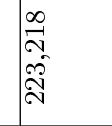 & $\begin{array}{l}\vec{J} \\
\stackrel{D}{0} \\
0 \\
-\end{array}$ & $\overleftrightarrow{z}$ \\
\hline 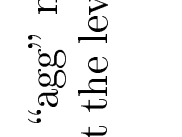 & בٓ & $\stackrel{0}{10}$ & $\begin{array}{l}0 \\
\infty \\
\infty \\
i \\
11\end{array}$ & $\mathscr{\sigma}$ & $\stackrel{9}{\rightarrow 19}$ & लै & $\begin{array}{l}\overrightarrow{1} \\
20 \\
21\end{array}$ & 茫 & $\vec{z} \mid \vec{z}$ & & 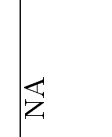 & 芝 & 岁 \\
\hline 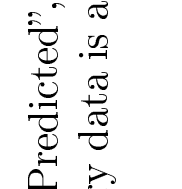 & 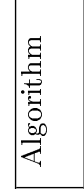 & $\sum_{0}^{Z}$ & 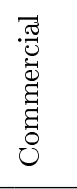 & 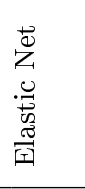 & 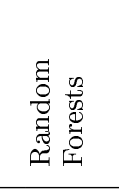 & 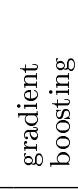 & 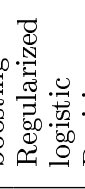 & 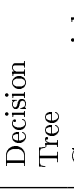 & 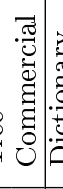 & & 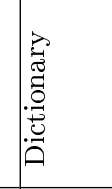 & 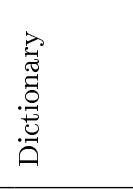 & 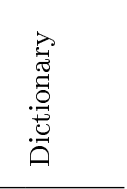 \\
\hline 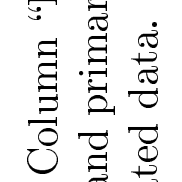 & 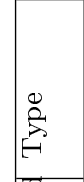 & 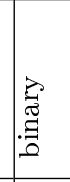 & $\begin{array}{l}0 \\
0 \\
0\end{array}$ & 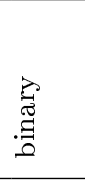 & 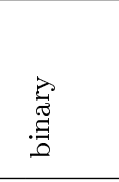 & $\begin{array}{l}\vec{\Xi} \\
\stackrel{\Xi}{\Xi} \\
\end{array}$ & : & 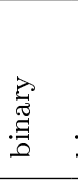 & 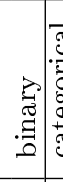 & & 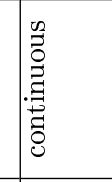 & 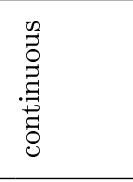 & 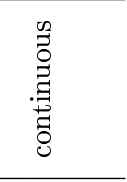 \\
\hline 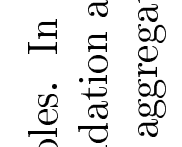 & 芯 & $\lambda$ & 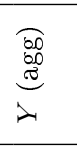 & 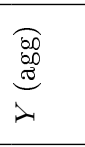 & 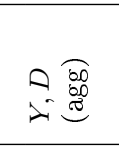 & $\lambda$ & $\lambda$ & 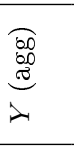 & 00 & A & $\lambda$ & $\theta$ & $\lambda$ \\
\hline 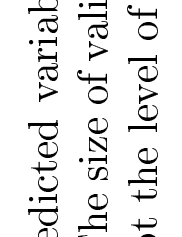 & $\lambda$ & 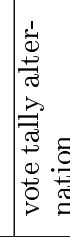 & 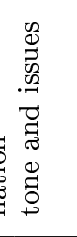 & 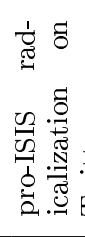 & 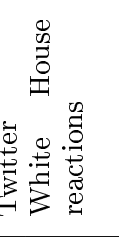 & 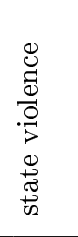 & $\begin{array}{l} \\
4 \\
0 \\
00 \\
00 \\
0 \\
0 \\
0 \\
0\end{array}$ & 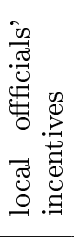 & 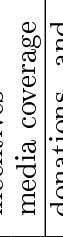 & 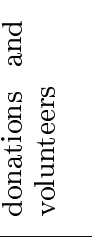 & 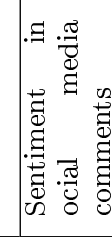 & 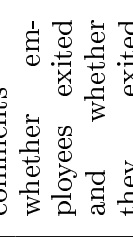 & 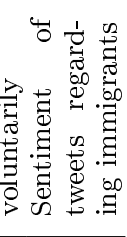 \\
\hline 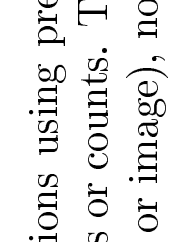 & 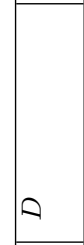 & 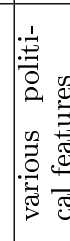 & & 峞 & 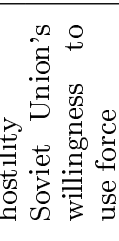 & 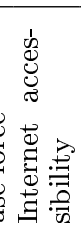 & 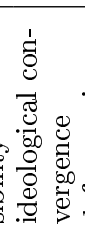 & 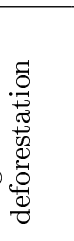 & 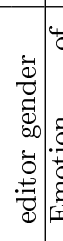 & 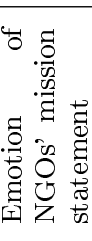 & 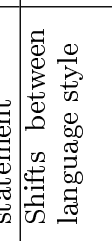 & 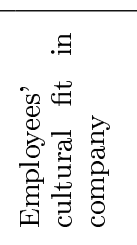 & 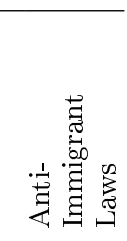 \\
\hline 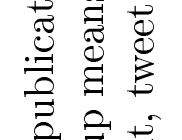 & 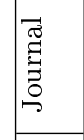 & $\frac{\pi}{4}$ & $\begin{array}{l}\frac{\pi}{w} \\
\tilde{2} \\
\frac{1}{4}\end{array}$ & 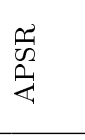 & $\begin{array}{l}n \\
\tilde{n} \\
2\end{array}$ & $\frac{n}{3}$ & $\begin{array}{l}\frac{1}{2} \\
2 \\
2\end{array}$ & $\stackrel{\text { I }}{\overrightarrow{0}}$ & $\begin{array}{lll}\frac{\pi}{2} \\
\frac{2}{4}\end{array}$ & 先 & 蛋 & 荌 & $\stackrel{2}{4}$ \\
\hline 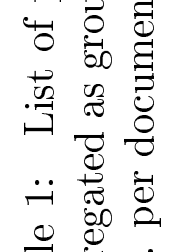 & 焉 & 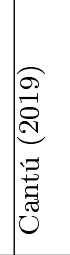 & 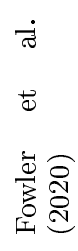 & 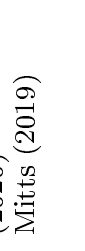 & 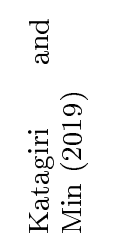 & 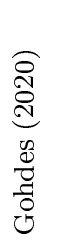 & 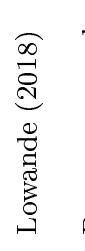 & 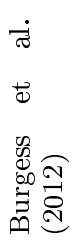 & 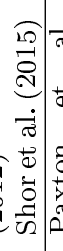 & 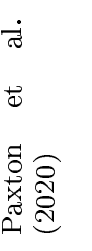 & 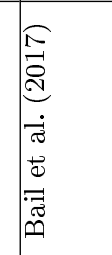 & 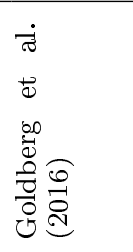 & 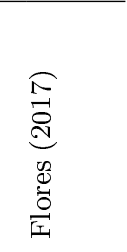 \\
\hline 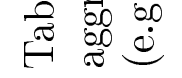 & $\dot{z}$ & - & N & $\infty$ & 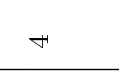 & 10 & 0 & $\wedge$ & $\infty^{\infty} p^{\circ}$ & & 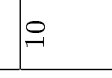 & $\exists$ & $\cong$ \\
\hline
\end{tabular}


tively correlated with the true values (Black et al., 2000). ${ }^{6}$ Therefore, using binary classification will lead to biased coefficient estimates, against common beliefs.

Last, some machine learning algorithms will first produce a continuous predicted probability and then coerce the probability into a binary classification based on a certain probability threshold (often 0.5 ). One conventional wisdom is that one should use continuous predicted probabilities instead of binary classification in next-step regression. ${ }^{7}$ For example, Studies 2 and 4 presented results using both predicted probabilities and binary classification as the dependent variables. However, using the predicted probability as variables, which is bounded between 0 and 1 , again violates the classical measurement error assumption and can lead to biased estimates of coefficients. We performed simulations to show that this is indeed the case (Appendix B).

\section{$3 \quad$ Regression with ML Classification Generally Leads to Attenuation Bias}

We first introduce four ML classification performance metrics, since they are directly related to the direction and size of biases in the coefficient estimates. For a binary random variable $X$ and its ML classification $\widetilde{X}$, two classification error rates are defined (the smaller the error, the better the algorithm):

- false positive rate: $F P R=P(\widetilde{X}=1 \mid X=0)$. High $F P R$ means high Type I error.

- false negative rate: $F N R=P(\widetilde{X}=0 \mid X=1)$. High $F N R$ means high Type II error. 
ML algorithms can also be evaluated using two performance metrics (the higher the performance metric, the better the algorithm):

- positive predicted value: $P P V=P(X=1 \mid \widetilde{X}=1)$. PPV is also commonly referred to as precision for positive class (1) in the machine learning literature.

- negative predicted values: $N P V=P(X=0 \mid \widetilde{X}=0)$. NPV is the precision for the negative class $(0)$.

$P P V$ and $N P V$ can be calculated if we know FPR and NPR. Thus, we will later generally refer the four metrics as error rates. ${ }^{8}$

\subsection{Intercept-only model}

We refer to the data that will be used to fit the next-step regression as the primary data. For primary data, we have only the prediction of the dependent variables, $\widetilde{Y}$, not its true values. ${ }^{9}$ We first discuss intercept-only regression (Equation 1), which is useful because the intercept $\alpha$ equals the mean of the binary outcome $(E(Y))$, and further equals the proportion of the positive class in the binary classification $(P(Y=1))$. An unbiased measure of intercept is critical for Studies 2 to 4 and 7 , in which researchers first produce binary classifications and then use the proportion of the positive class (or count, which is the proportion multiplied by the number of observations) as a variable in next-step regression (Column "agg"). For instance, Mitts (2019) (Study 3) examines explanations for online radicalization and support for ISIS on Twitter. She first used ML algorithms to predict whether each tweet was radical or not, and then used the number of radicalized tweets from each user (i.e. count) as the dependent variable.

$$
Y=\alpha+\epsilon, E(\epsilon)=0,
$$


Unfortunately, the naive estimate of the intercept does not equal the true $\alpha$. Put differently, the positive class proportion calculated from the ML classification $(P(\widetilde{Y}=1))$ does not equal the true proportion (Hausman et al. (1998)): ${ }^{10}$

$$
\begin{aligned}
\hat{\alpha}^{\text {naive }} & =P(\tilde{Y}=1)=E(\tilde{Y})=\alpha(1-F N R-F P R)+F P R, \text { or } \\
\alpha & =E(Y)=P(Y=1)=\frac{\hat{\alpha}^{\text {naive }}-F P R}{1-F N R-F P R}=\frac{P(\tilde{Y}=1)-F P R}{1-F N R-F P R}
\end{aligned}
$$

Simple algebra shows that $\hat{\alpha}^{\text {naive }}$ overestimates the true $\alpha$ when $\hat{\alpha}^{\text {naive }}>\frac{F P R}{F P R+F N R}$. Equation 2 will be particularly helpful in obtaining the correct measure of the proportions if one uses proportion or counts as the outcome variable, as in Studies 2 to 4 and 7.

\subsection{ML classification as outcomes}

We add independent variables to next-step regression models:

$$
\begin{array}{r}
Y=\alpha+\beta D+\gamma W+\epsilon, E(D \mid \epsilon)=0, \text { linear regression } \\
P(Y=1 \mid D, W)=\operatorname{logit}^{-1}(\alpha+\beta D+\gamma W), \text { logistic regression, }
\end{array}
$$

where $\beta$ is the key parameter of interest, $D$ is a focal independent variable, and $W$ is a matrix containing additional control variables. The naive regression coefficients, which are obtained by regressing predicted values $(\widetilde{Y})$ on independent variables, are

denoted with a "naive" superscript (e.g. $\hat{\beta}^{\text {naive }}$ ). We need two additional assumptions: 
Assumption $1 \quad \tilde{Y} \Perp D, W \mid Y$. The predictions are independent of other variables in the regression, given their true values.

Assumption 1 is known as nondifferential measurement error (Carroll et al., 2006). It is often the default assumption in the measurement error literature (but see Imai and Yamamoto (2010)). Assumption 1 means that the prediction error is independent of the other variables. In other words, the prediction error should not be systematically greater at certain values of other variables in the regression. Take Cantú (2019) as an example. One of the independent variables that he used was whether members of the opposition party were present. If fraudsters worked more diligently to alter ballots when the opposition was not present, such that it made it harder for the classifier to detect the alterations, Assumption 1 would be violated.

Without external validation data, researchers sometimes use theory or domain knowledge to justify nondifferential measurement error (e.g. if the true and predicted variable occurred first and the outcome is measured later in time) (Carroll et al., 2006, p. 36). With external validation data, Assumption 1 can be statistically tested (more detail on external validation datasets is available in Section 4). Scholars can perform statistical tests (e.g. t-tests, or the regression of prediction errors on other variables) to see whether prediction errors are significantly different across strata set by the outcome or other control variables. Some studies in Table 1 (e.g. Study 2 and 3) have provided statistical evidence for the validity of Assumption 1 in their research; it is recommended that other researchers also apply this practice.

\section{Assumption $2 \quad F P R+F N R<1$}

Assumption 2 is trivial because random guesses will produce FPR and FPR equal to 0.5. As long as the ML algorithm is slightly better than random guesses, Assumption 2 will be satisfied. 
Linear regression Hausman et al. (1998) first shows that given Assumptions 1 and 2, if linear regression is used (i.e. the linear probability model), ${ }^{11}$ and if there is no control variable (i.e. $W$ does not exist), naive regression attenuates the true slope coefficient:

$$
\hat{\beta}^{\text {naive }}=\beta(1-F N R-F P R) .
$$

When ML classifications make more errors, $F N R$ and $F P R$ will be greater, and the attenuation bias will be more severe. The scale of attenuation $1-F N R-F P R$ is known as Youden's Index. It can be visualized as an ROC curve (receiver operating characteristics curve) (Appendix C).

The standard error estimates of the naive coefficients will also be smaller than the true values, also by a factor of $1-F N R-F P R$. This makes sense because naive regression treats ML predictions as error-free, whereas in reality they contain uncertainty which will boost the standard error estimates. In other words, naive regression not only results in a smaller effect size estimate, but also underestimates true standard errors and thus could lead to false discovery.

Logistic regression For logistic regression, although naive coefficients are typically attenuated, there is no closed-form formula linking $\beta$ and $\hat{\beta}^{\text {naive }}$. However, there is a closed-form formula linking the marginal effect estimates: ${ }^{12}$

$$
\frac{\partial P(\widetilde{Y}=1 \mid D)}{\partial D}=(1-F N R-F P R) \frac{\partial P(Y=1 \mid D)}{\partial D} .
$$




\subsection{ML classification as an independent variable}

Next, we discuss cases in which researchers use a linear regression to regress $Y$ on ML predicted $\widetilde{D}$. We adapted the two assumptions as follows.

Assumption 1A $\quad \widetilde{D} \Perp Y, W \mid D$. The predictions are independent of other variables in the regression, given their true values.

\section{Assumption 2A $\quad P P V+N P V>1$}

Aigner (1973) first proved that given Assumptions 1A and 2A, and where there is no control variable, the naive estimate will lead to attenuation bias (because $1<$ $P P V+N P V<2):$

$$
\hat{\beta}^{\text {naive }}=\beta(P P V+N P V-1) .
$$

Finally, if the next-step regression contains additional control variables $W$, there is no simple closed-form formula linking $\hat{\beta}^{\text {naive }}$ and its true value $\beta$, in both predicted independent and dependent variable cases. There have been detailed investigations of the bias of $\hat{\beta}^{\text {naive }}$ when $W$ is present (Black et al., 2000; Meyer and Mittag, 2017). The conclusions of these studies are that unless the classification error has an extremely high correlation with a control variable, $\hat{\beta}^{\text {naive }}$ still attenuates the true $\alpha .{ }^{13}$ Our own numerical simulations show similar observations (Section 6).

Our review of the errors-in-variables models reveal the direction and sizes of the biases of coefficient estimates (or marginal effects) resulting from the use of binary ML classification in next-step regression. Although these results have been established for years, strikingly, no previous study has pointed out that ML classification error can be considered as a type of measurement error. Hence, all published studies in Table 1 suffer from the biases by using ML classifications as they were error-free. 


\section{Estimating error rates from validation data}

From our review, it is easy to see that if we know the error rates, there is hope to recover the true coefficients. However, by definition, we do not have true labels of the primary data, which is the data that researchers will ultimately apply ML algorithms to and use the predictions to run next-step regressions. Hence we cannot calculate errors for the primary data directly. We next discuss how to estimate the error rates.

\subsection{Creating validation data}

We require that researchers have validation data. The validation data includes both predictions and true values. The critical assumption about the validation data is:

Assumption 3. The validation data and the primary data are randomly sampled from the same population, and the validation data are never seen by machine learning algorithms.

Validation data that satisfy Assumption 3 can be obtained using two approaches:

Approach 1: From training data In the most commonly seen situation, researchers randomly sample a subset of their data, label this subset, and use it as training data for the ML algorithm. The validation data can then be obtained by holding back a proportion (e.g. 5 or 10\%) of the training data (the rest will be used to train algorithms). This approach follows the standard out-of-sample validation strategy in supervised machine learning algorithms, and one can check many mature review articles on how to create such a validation dataset (Grimmer and Stewart, 2013). Studies 1 to 6 in Table 1 followed this approach.

Approach 2: From primary data In this case, there are no training data because one uses dictionary methods or applies off-the-shelf pre-trained supervised mod- 
Truth

\begin{tabular}{c|c|c|c|}
\cline { 3 - 4 } \multicolumn{1}{c|}{} & \multicolumn{1}{c|}{0} & 1 \\
\cline { 2 - 4 } Prediction & TN (true negative) & FN (false negative) \\
\cline { 2 - 4 } & 0 & FP (false positive) & TP (true positive) \\
\cline { 2 - 4 } & &
\end{tabular}

Table 2: Confusion Matrix (defined on validation data)

els (this was the case in Studies 7 and 8). Or, one has training data, but they do not follow the same distribution as the primary data, so Assumption 3 is not satisfied. ${ }^{14}$ In these cases, one can create validation data from the primary data:

1. select a small random subset from the primary data

2. manually label the data to create "ground-truth" labels for the predictions.

Regardless of which approach researchers have taken, validation datasets are typically small, much smaller than the primary dataset. As one can see in Table 1 (column $\left.n_{\text {val }}\right)$, the number of observations in the validation dataset can be as small as 150 , and is typically less than 1,000 . The minimum ratio between primary and validation dataset size is 87 .

\subsection{Point estimates of error rates}

With validation data that satisfy Assumption 3, one can use the classification errors of the validation data, which can be calculated, to approximate the classification errors when one applied ML algorithm on the primary data (Hastie et al., 2009, Chap. 7.2). Specifically, one can tabulate the prediction and the true labels in the confusion matrix as described in Table 2. $T P, T N, F P, F N$ refer to the number of true positives, true negatives, false positives, and false negatives in the validation data, respectively. We can then estimate $F P R, F N R, P P V$ and $N P V$ as: 
- $\widehat{F N R}=\frac{F N}{F N+T P} ; \widehat{F P R}=\frac{F P}{F P+T N}$,

- $\widehat{P P V}=\frac{T P}{T P+F P} ; \widehat{N P V}=\frac{T N}{T N+F N}$

However, if Assumption 3 is not met, and in particular if one uses the same data both for training ML algorithms and for estimating error rates, $\widehat{F N R}$ and $\widehat{F P R}$ will underestimate the FNR and FPR of the primary data. We discuss estimating error rates in more detail in Appendix D.

\subsection{Variance of error rates}

Because the validation dataset is often small, the error rate estimates may have high variances. Past literature on the errors-in-variables model has rarely considered how to estimate variance of error rates, not to mention how to incorporate the variance into correcting next-step regression coefficients.

This article borrows the knowledge of the computer science community to estimate the variances of error rates. The first approach uses Bootstrap methods: resampling the validation data, calculating error rates for each resample, and obtaining a sampling distribution of error rates (Bisani and Ney, 2004; Sakai, 2006).

The second approach takes a Bayesian perspective. Goutte and Gaussier (2005) suggest that $F N$ follows a binomial distribution because $F N$ is the number of times false negative cases occur out of all positive cases $(F N+T P)$, and the probability of occurrence is $F N R$. The distribution of $F P, T P$, and $T N$ also follows binomial distributions:

$$
\begin{aligned}
& \text { - } F N \sim \operatorname{Binomial}(F N+T P, F N R) \\
& \text { - } F P \sim \operatorname{Binomial}(F P+T N, F P R) \\
& \text { - } T P \sim \operatorname{Binomial}(T P+F P, P P V)
\end{aligned}
$$


- $T N \sim \operatorname{Binomial}(T P+F N, N P V)$

A natural choice for the prior of binomial parameters is its conjugate, a Beta distribution. We chose a non-informative prior: the uniform prior $(a=b=1) \cdot{ }^{15}$

- $F N R, F P R, P P V, N P V \sim \operatorname{Beta}(a, b)$

Certainly, the ultimate goal is to incorporate the variance of error rates into the variance estimates for next-step regression coefficient, which is a gap in the literature. Next, we propose new solutions in Sections 5.4 and 5.5.

\section{Estimators}

\subsection{Using validation data to run regression}

When validation data exist, the simplest approach to obtaining an unbiased and consistent coefficient estimate is to use the true labels in the validation data to run regression. We denote the slope coefficient as $\hat{\beta}^{\text {val }}{ }^{16}$ Although $\hat{\beta}^{\text {val }}$ is in theory unbiased and consistent, it may suffer from finite-sample bias, and the variance estimate will be high due to the small sample size of the validation dataset. Moreover, using a small validation dataset defies the very reason for researchers to use "big data": to investigate heterogeneous effects, such as fine-grained regional variations of effects (Salganik, 2019; Fowler et al., 2020). Last, many studies (e.g. Studies 2-4) cannot be performed because these studies further aggregate their predictions by groups and they cannot achieve a meaningful number of observations within each group if they used validation data to run their regressions. ${ }^{17}$

In practice, we find that regression on validation datasets can be used for two purposes. First, one can always use $\hat{\beta}^{\text {val }}$ as a robustness check for the point estimates. 
This can become particularly useful if one suspects that Assumption 1 has been seriously violated (more in Section 6.4). Second, because the confidence intervals of $\hat{\beta}^{v a l}$ are wide, it should have an very high chance to cover the true coefficients. Hence, we can instruct our estimators to find coefficient estimates within the $95 \%$ confidence interval of the regression using validation data $\left(v_{\text {lower }}, v_{\text {upper }}\right)$. This approach can effectively rule out some bad estimates very easily (Section 5.3 to 5.5).

\subsection{Analytical correction}

In the simplest case- linear regression without additional control variables - the coefficients can be consistently estimated based on Equations 2 to 7 . Because researchers will almost always add control variables, we suspect that they will not use analytical correction to obtain slope estimates. We have therefore included the detailed formula in Appendix E. ${ }^{18}$

However, when researchers aggregate their binary predictions into counts or proportions, a particular analytical correction formula-Equation 2-can be used to obtain the group-specific counts or proportions. We found this formula useful when replicating Study $2-4$.

\subsection{MLE estimators}

The more general estimator is based on MLE. The key is to use Bayes' rule to express the observed data likelihood as a function of the true data likelihood and the estimated classification error rates. Take the example of using ML classification as the outcome variable. Bayes' rule suggests that observed data probability $P(\widetilde{Y}=1 \mid D, W)=$ $F P R+(1-F P R-F N R) P(Y=1 \mid D, W)$. The true data probability $P(Y=1 \mid D, W)$

further links with the true coefficients through an inverse-logit function (Equation 4). 


\section{ML binary classification as the outcome variable in for logistic regression}

(Hausman et al. (1998); derivation in Appendix H):

$$
\hat{\alpha}^{M L E}, \hat{\beta}^{M L E}, \hat{\gamma}^{M L E}=\underset{\alpha, \beta, \gamma}{\arg \max } \log L,
$$

where $\log$-likelihood $\log L$ is

$$
\begin{aligned}
& \log L=\sum_{i=1}^{n} \tilde{Y}_{i} \ln \left[F P R+(1-F N R-F P R) \cdot p_{i}\right]+\left(1-\tilde{Y}_{i}\right) \ln \left[1-F P R-(1-F N R-F P R) \cdot p_{i}\right], \\
& \quad \text { and } p_{i}=P\left(Y_{i}=1 \mid D_{i}, W_{i}\right)=\operatorname{logit}^{-1}\left(\alpha+\beta D_{i}+\gamma W_{i}\right) .{ }^{19}
\end{aligned}
$$

The standard error of the regression coefficients can be obtained by using the inverse of the Hessian matrix, following the typical procedures for MLE estimation.

Similarly, we can derive the MLE estimator for cases in which ML binary classification is used as the independent variable in linear regression. Aigner (1973) has already pinned down this idea, but did not write down the exact formula. Due to space limitations, the full derivation is provided in Appendix I.

Bounding parameter estimates In our simulations, we found that the above MLE works perfectly if Assumptions 1 to 3 are met. However, when Assumption 1 is violated, and the prediction error is thus correlated with other independent variables, the raw MLE listed above can sometimes give very bad estimates. Fortunately, there is an easy way to avoid these extremely bad estimates. We can force the optimization algorithm to find coefficient values within the confidence interval set by the regression with validation data. In practice, we find that this simple restriction avoids some oddly large estimates resulting from violation of Assumption 1 or even more extreme situations. 
The analytical correction and the MLE estimator treat $F P R / F N R$ as fixed. Therefore, they may underestimate the true standard errors for the next-step regression coefficients. Next, we propose two new estimators-Bootstrap and Bayesian estimators - which follow naturally from our discussions of estimating variance of error rates in Section 4.3.

\subsection{Bootstrap estimator}

The Bootstrap estimator resamples the validation data, calculates resampled error rates, and uses the MLE estimator above to calculate resampled estimates of coefficients. Formally:

1. sample $n_{v a l}$ integers from 1: $n_{\text {val }}$ with replacement. Denote the sampled integers as $S_{k}$.

2. obtain resampled validation dataset $V_{k}=\left\{Y_{i}, \widetilde{Y}_{i} \mid i \in S_{k}\right\}$ (for predicted outcome), or $V_{k}=\left\{D_{i}, \widetilde{D_{i}} \mid i \in S_{k}\right\}$ (for predicted independent variable).

3. calculate resampled error rates, $F N R_{k}, F P R_{k}$ ((for predicted outcome), or $P P V_{k}, N P V_{k}$ (for predicted independent variable) using the resampled validation dataset $V_{k}$.

4. use the the resamped error rates in the MLE estimator to obtain coefficients.

Steps 1 - 4 will be repeated $K$ times $(k \in 1: K) ; K$ should be a reasonably large integer. Then the mean, standard deviation, or quantile function can be applied to obtain an estimation of the regression coefficients, their standard errors and confidence intervals, based on sets of $K$ resampled coefficient estimates. 


\subsection{Bayesian estimator}

The Bayesian estimator for regression coefficients uses the Bayesian estimator for error rates, and the likelihood function in MLE described above. If ML classification is used as the outcome variable in a logistic regression, the full MCMC sampling procedure is as follows.

- $F N R, F P R \sim \operatorname{Beta}(1,1)$

- $F N \sim \operatorname{Binomial}(F N+T P, F N R) ; F P \sim \operatorname{Binomial}(F P+T N, F P R)$

- $1 / \sigma^{2} \sim$ half-Cauchy $(0,2.5) ;^{20}$

- $\alpha, \beta, \gamma \sim$ Truncated-Normal $\left(0, \sigma^{2} ; v_{\text {lower }}, v_{\text {upper }}\right)$; The use of truncated normal distribution restricts parameter estimates to a range within $\left(v_{\text {lower }}, v_{\text {upper }}\right)$, i.e. the $95 \%$ confidence intervals from coefficient estimates of validation dataset regression.

- $P(Y=1 \mid D, W)=\operatorname{logit}^{-1}(\alpha+\beta D+\gamma W) ;^{21}$

- $P(\widetilde{Y}=1 \mid D, W)=F P R+(1-F P R-F N R) P(Y=1 \mid D, W)$;

- $\widetilde{Y} \sim \operatorname{Bernoulli}(P(\widetilde{Y}=1 \mid D, W))$.

We also derived a Bayesian estimator for cases in which ML classification is used as an independent variable. Full details are provided in Appendix J.

\section{Simulations}

We demonstrate the proposed estimators by examining a series of Monte Carlo simulations. Because we know the true values of the coefficients in the simulations, we can determine whether the proposed estimators can identify the true values. 


\subsection{Simulation 1: ML classification as outcome}

The data generating process (DGP) was as follows:

- $D, W \sim N(0,1)$;

- $Y^{*}=\operatorname{logit}^{-1}(\alpha+\beta D+\gamma W)$, where $\alpha=0.5, \beta=1$, and $\gamma=1$;

- $F N R=0.1$ and $F P R=0.1$;

- $Y \sim \operatorname{Bernoulli}\left(Y^{*}\right)$

- The predicted $\widetilde{Y}$ was generated from the true $Y$ :

- If $Y=1$, we made it 0 with probability $F N R$.

- If $Y=0$, we made it 1 with probability $F P R$.

We generated 9,100 data points from the DGP and then randomly selected 100 as the validation data; the remaining 9000 made up the primary sample. We intentionally designed a small validation dataset to assess whether the small size impacted our estimators. The size of the primary dataset was chosen because the ratio between primary and validation data was roughly the minimum ratio between the primary and validation dataset sizes given in Table 1. For both Bayesian and Bootstrap estimators, we took 10,000 MCMC/resampling draws. ${ }^{22}$ The results were averaged over 50 sampled datasets to smooth the estimates. We note that Bayesian estimator is considerably slower than the rest estimators.

Figure 1 suggests that naive logistic regression attenuates all coefficients. ${ }^{23}$ MLE, Bayesian, and Bootstrap estimators recover the true coefficient. Figure 2 plots the posterior (resampling) distribution of $F N R$ and FPR from Bayesian (Bootstrap) estimators. The Bayesian estimator has a wider credible interval than the MLE estimator, but it has a narrower confidence interval than the Bootstrap estimator. ${ }^{24}$ 


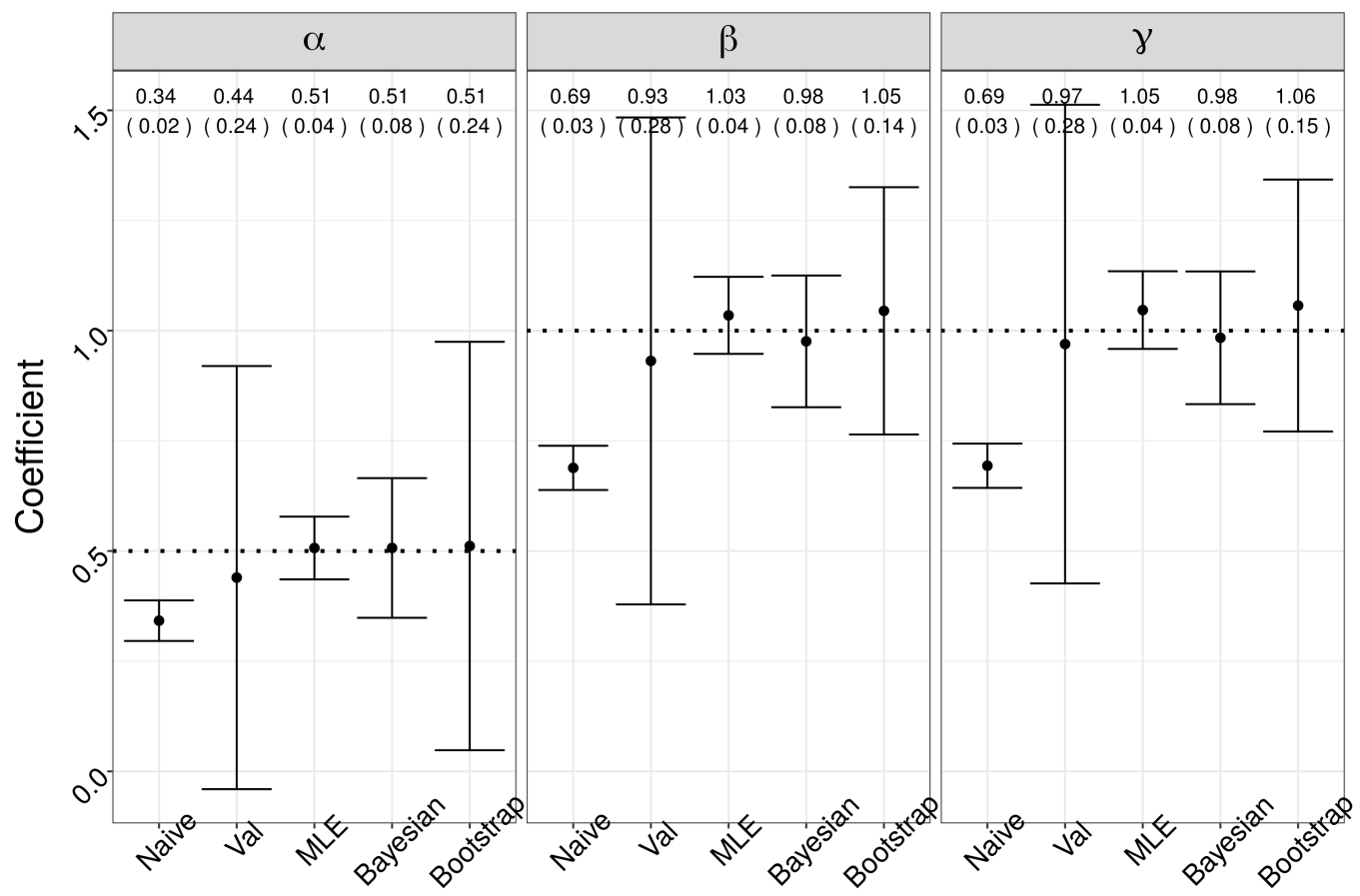

Figure 1: Estimated regression coefficients with 95\% confidence (credible) intervals when ML classification is used as the outcome in logistic regression. The point estimates and their standard errors are listed on the top of each group. The true value of each coefficient is plotted as a dotted horizontal line. 

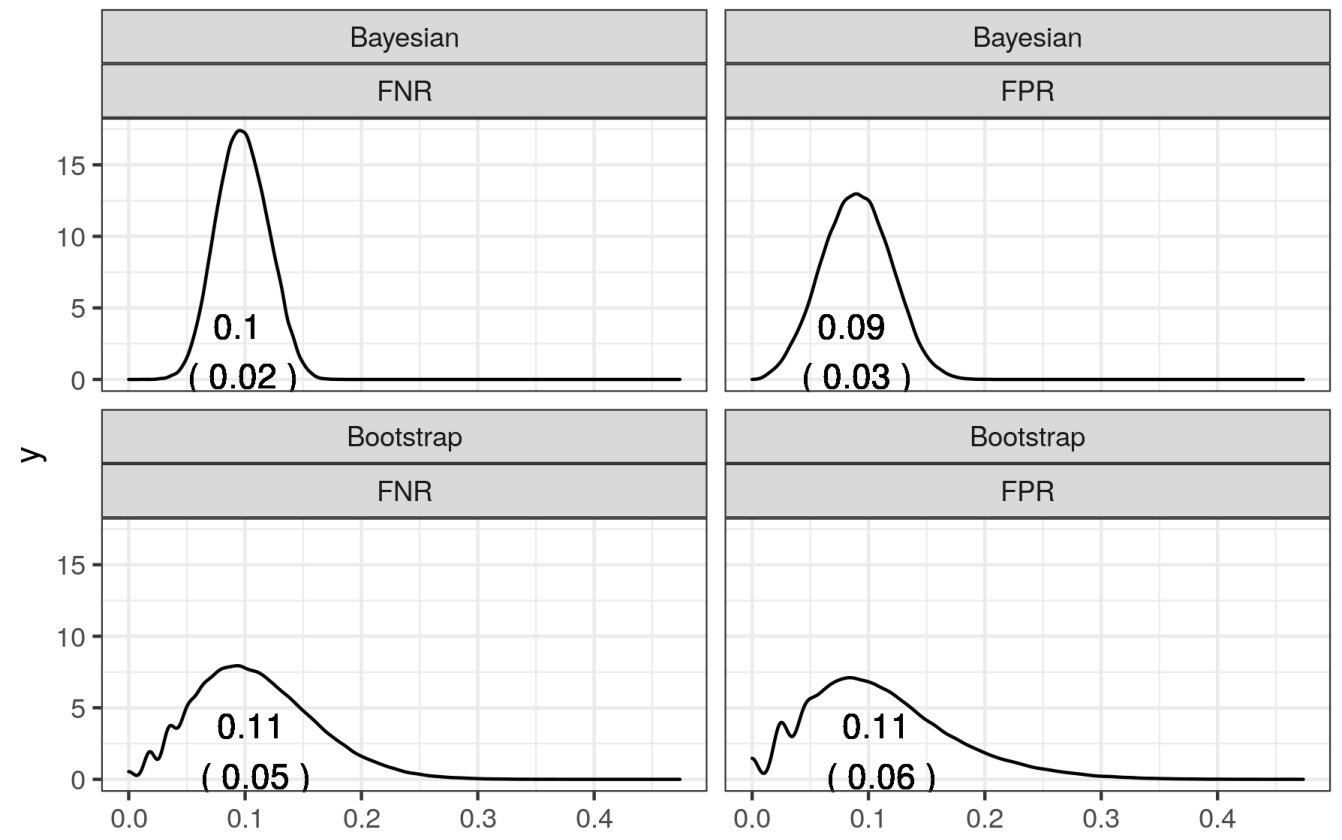

Figure 2: Posterior/resampling distribution of FNR and FPR when ML classification is used as the outcome in logistic regression. Grey vertical line is the mean. The point estimates and their standard errors are spelled out in each panel. 


\subsection{Simulation 2: ML classification as an independent variable}

The data generating process (DGP) is as follows:

- $D=\mathbf{I}\left(D^{*}>0\right)$, where $\mathbf{I}$ is an indicator function and $D^{*} \sim N(0,1)$;

- $W \sim N(0,1)$ is an additional control variable independent of $D$;

- $Y=\alpha+\beta D+\gamma W+\epsilon$, where $\alpha=0.5, \beta=1, \gamma=1$, and $\epsilon=N(0,1)$;

- $F N R=0.1$ and $F P R=0.1 .^{25} P P V$ and $N P V$ are both 0.895 .

- The predicted $\widetilde{D}$ was generated from the true $D$ :

- If $D=1$, we made it 0 with probability $F N R$.

- If $D=0$, we made it 1 with probability $F P R$.

Again, we generated 9,100 data points from the above process and randomly selected 100 as validation data. The remaining 9,000 made up the primary sample. Figure 3 suggests that naive regression inflated the intercept $(\alpha)$ by $20 \%$ and attenuated the slope coefficient for the predicted variable by $20 \%(\beta)$. The MLE, Bayesian, and Bootstrap estimators recovered the true coefficients and were much more efficient than the regression using only validation data. Figure 4 plots the posterior/resampling distribution of $P P V$ and $N P V$ under the Bayesian and Bootstrap estimators.

An interesting observation is that when ML classification is used for outcome variables, the Bayesian estimator is more efficient than the Bootstrap estimator. However, when ML classification is used as the independent variable, the Bootstrap estimator is more slightly more efficient. These observations are shared by other simulations we will discuss below. Why this is the case? We find that the reason lies in the scale of error rates. $F P R, F N R$ is typically much smaller than $P P V / N P V$. Bayesian estimates 
of variance of error rates vary by the scale of each quantity, but Bootstrap resampling gives a similar amount of variances for all four quantities. For ML-predicted outcome variables, FNR and FPR are relevant for biases. Bayesian estimates of FPR and $F N R$ have a smaller variance estimates of the error rates, which resulted in smaller standard errors of the coefficient from the Bayesian estimators. ${ }^{26}$

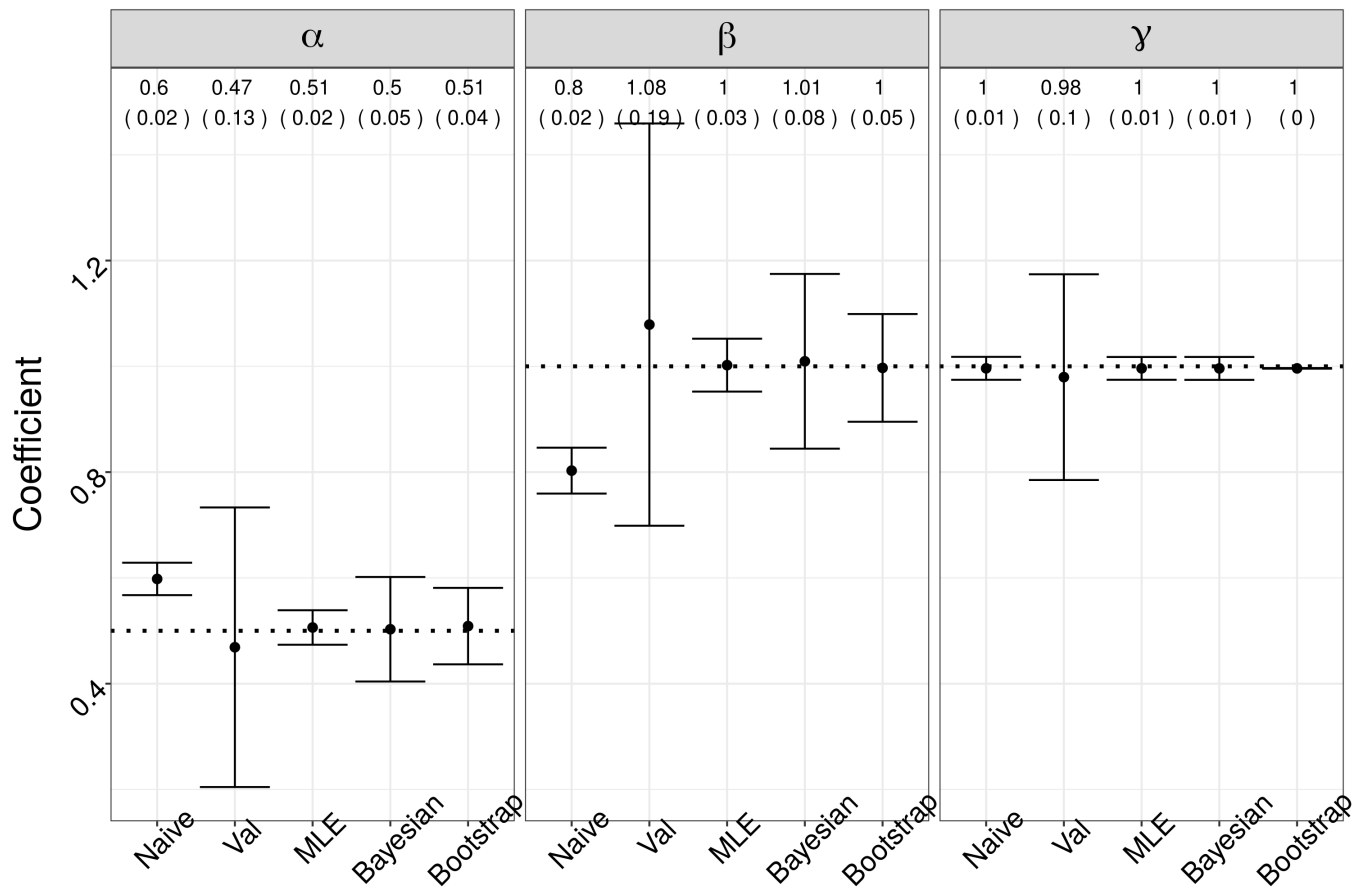

Figure 3: Estimated regression coefficients with 95\% confidence (credible) intervals when ML classification is used as the independent variable in linear regression. The point estimates and their standard errors are listed at the top of each group. The true value of each coefficient is plotted as a dotted horizontal line.

\subsection{Size of validation data}

A critical decision that researchers must make is choosing the size of the validation dataset. A larger validation dataset will reduce the variance of the error rates and therefore the standard errors of coefficients. However, a larger validation dataset 

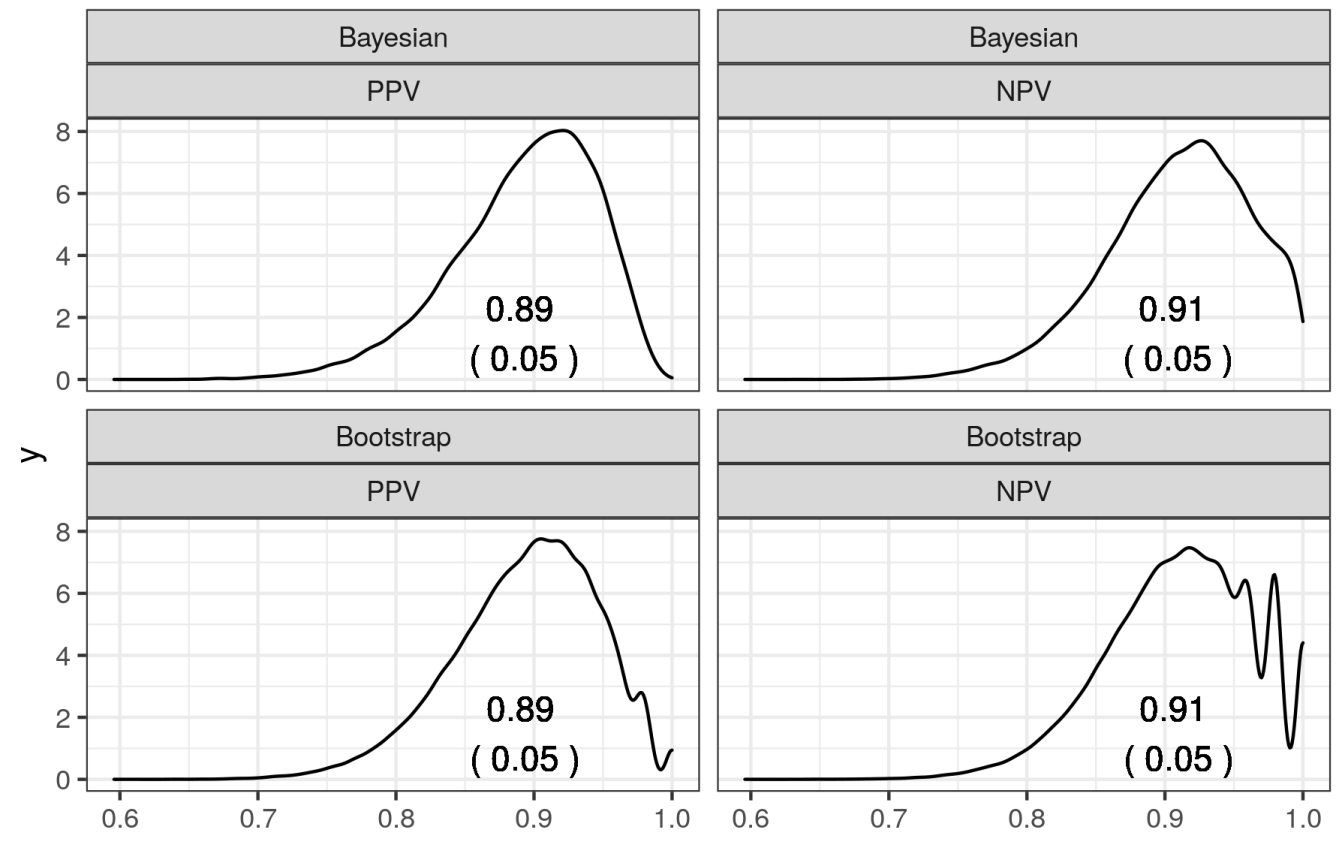

Figure 4: Posterior/resampling distribution of PPV and NPV when ML classification is used as the independent variable. Grey vertical line is the mean. The point estimates and their standard errors are spelled out in each panel. 


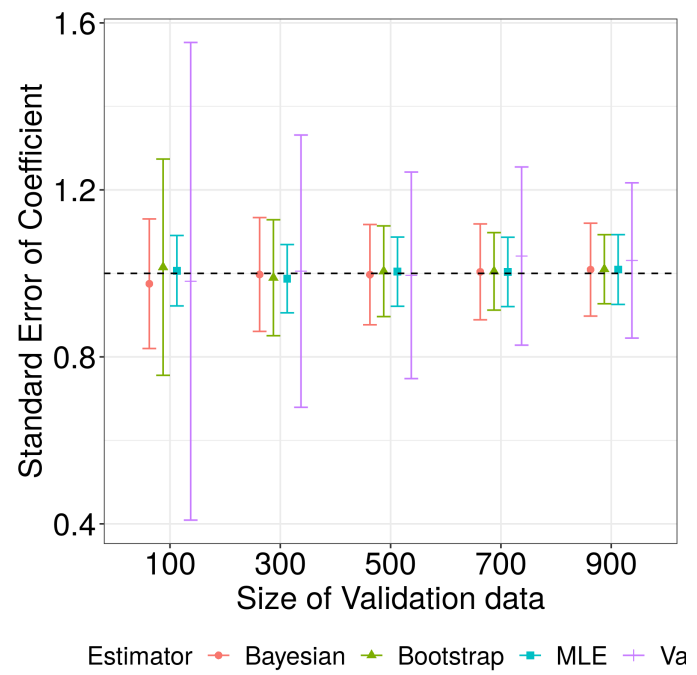

(a) Predicted outcome.

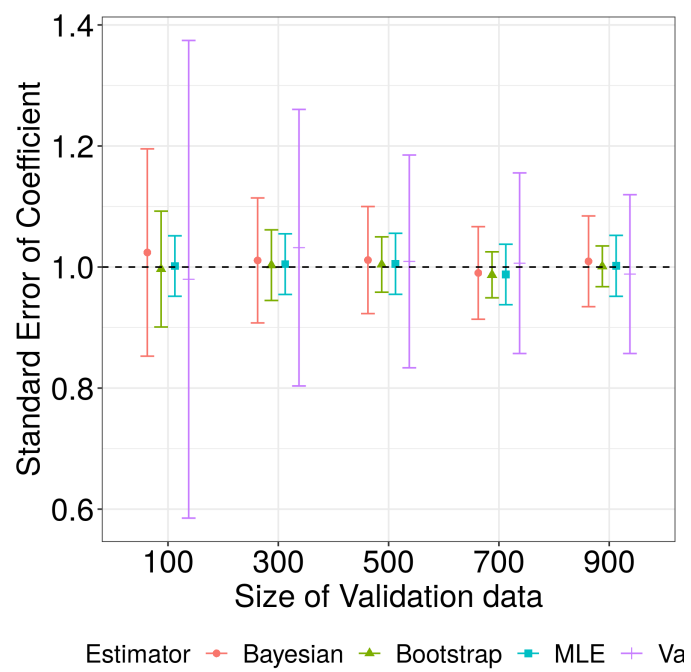

(b) Predicted independent variable.

Figure 5: Coefficient estimates by validation dataset size.

will also cost more money and time to create. ${ }^{27}$ To evaluate the trade-off between statistical efficiency and cost, we varied the size of the validation dataset between 100 and 900 with a distance of 200 , while keeping the other variables the same, in Simulations 1 and 2. Figure 5 suggests that a validation dataset of size 100 is too small. As long as the size of the validation dataset reaches 500, further increases in the size of the validation dataset will no longer result in a major reduction in the width of the confidence interval.

\subsection{Correlated ML classification and control variables}

Assumption 1 (1A) assumes that the prediction error is uncorrelated with other variables in the regression. How does the violation of Assumption 1 impact our estimators? We changed Simulation 1 (2) to let the prediction error $\widetilde{Y}-Y(\widetilde{D}-D)$ and the independent variable $D(W)$ be correlated (detail of this procedure is provided in Appendix M). ${ }^{28}$

Figure 6a shows that if the dependent variable is predicted and the prediction 


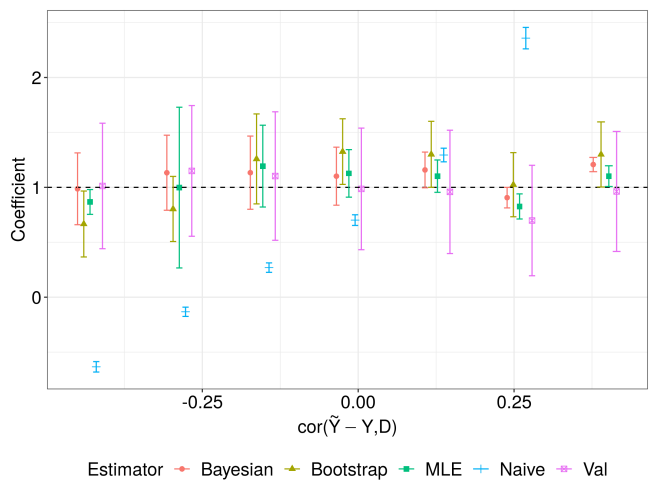

(a) Predicted outcome.

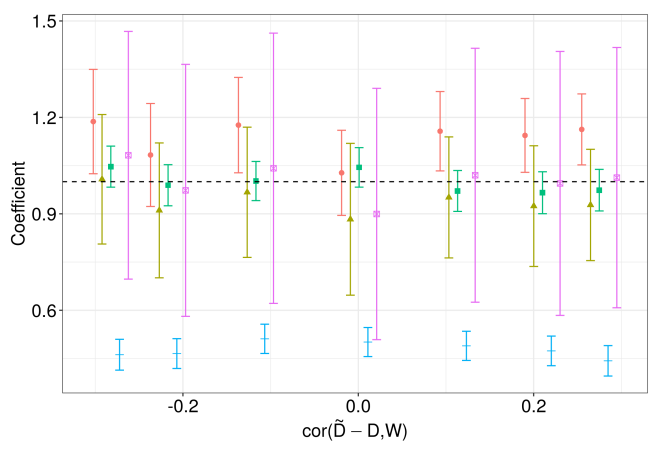

Estimator - Bayesian + Bootstrap $=$ MLE + Naive $*$ Val

(b) Predicted independent variable.

Figure 6: Estimated regression coefficients with 95\% confidence (credible) intervals. The true value of each coefficient is plotted as a dotted horizontal line.

error is correlated with the independent variable $D$, then naive estimates lead to large biases. The size of the bias increases as the scale of the correlation increases. The bias can become so large that even the sign of coefficient estimates will be inverted. The presence of correlated observed confounders also leads to biases for all other estimators, compared with Simulation 1 where there is no correlation. However, the MLE, Bootstrap, and Bayesian estimators generally provide much better estimates than naive regression. ${ }^{29}$

Figure $6 \mathrm{~b}$ shows that if the independent variable $D$ is predicted, and the prediction error is correlated with another independent variable $W$ in the regression, then naive estimates consistently result in a large and fixed bias. The bias scale is larger than the bias of the naive regression of Simulation 2 when the prediction error and $W$ are not correlated. MLE, Bootstrap, and Bayesian estimators are biased, but at a smaller scale than naive estimates.

Next, we explore a more challenging case: the prediction error is correlated with an unobserved confounder, which results in omitted variable bias (OVB). ${ }^{30}$ Here, both the ML classification error and the OVB will bias the regression coefficients. ML clas- 
sification errors generally lead to attenuation biases, whereas OVB can attenuate or inflate coefficient estimates. The inconsistency of the direction of the bias suggests that sometimes the ML classification error and the OVB will work in the same direction and thus produce a larger attenuation bias; sometimes, they will work in the opposite direction and produce a good estimate by an odd coincidence. ${ }^{31}$ Results are shown in Figure 7.

Comparing Figure $7 \mathrm{a}$ with $6 \mathrm{a}$, we can see that the addition of OVB generally make coefficients more biased. Bayesian and MLE estimators produce much better estimates than naive estimates. Bootstrap estimators have wide confidence intervals. Figure $7 \mathrm{~b}$ shows a similar observation for cases of predicted independent variables; naive estimates are still the worst. This time Bayesian estimators have larger biases than MLE and Bootstrap estimators.

Figures 6 and 7 show that the violation of Assumption 1 (1A) impacts the proposed estimators and results in relatively large biases compared to cases in which the Assumptions are met. These are limitations of the current proposed estimators. However, all of the proposed estimators are typically much better than naive estimates, which can even result in erroneous signs. Hence, even if one suspects that Assumption 1 (1A) has been violated, one still should not use naive regression.

From Figure 6 and 7, we can also observe that regression using validation data generally results in better point estimates than naive regression, although it also suffers from finite-sample bias and results in the widest confidence interval. When one suspects that Assumption 1 has been violated, our recommendation is to compare our estimators with the regression using validation dataset. If the two produce markedly different point estimates, researchers can collect more validation data and see if the difference is due to the finite-sample bias of the regression on validation data only, or due to the bias resulting from the violation of Assumption 1. 


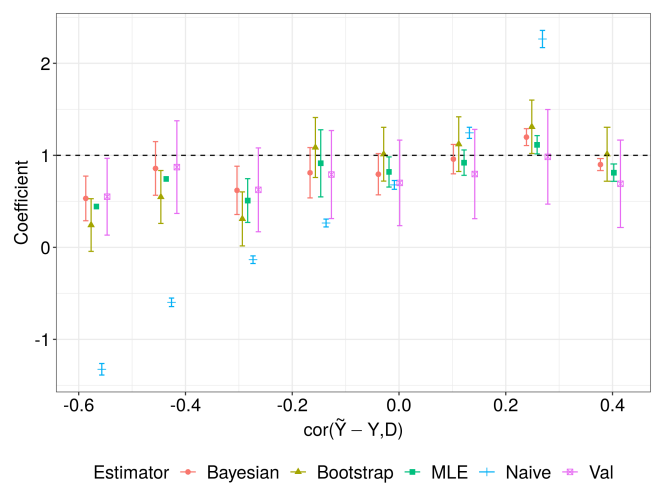

(a) Predicted outcome. ${ }^{32}$

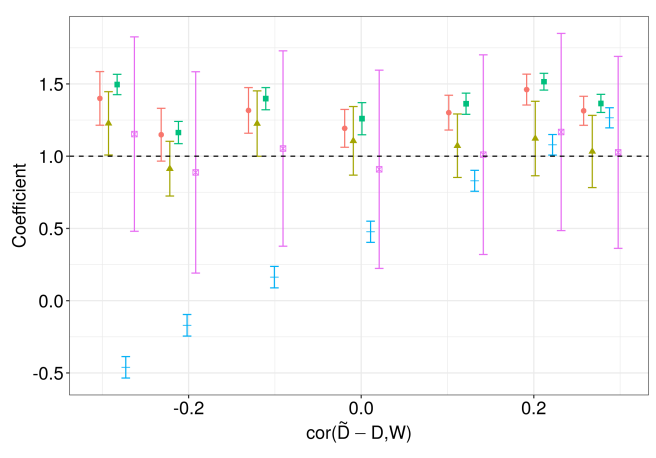

Estimator - Bayesian $\neq$ Bootstrap $=$ MLE + Naive $*$ Val

(b) Predicted independent variable.

Figure 7: Estimated regression coefficients with 95\% confidence (credible) intervals. The true value of each coefficient is plotted as a dotted horizontal line.

Finally, an anonymous reviewer is concerned about the possibility that bias due to violation of Assumption 1 will increase when the size of the primary dataset increases. We repeated the simulations in Figure 6 and 7, but increased the size of the primary data up to a million. Due to space limitations, the results are presented in detail in Appendix N.2. We do not find support for the hypothesis that increasing the size of the primary dataset will result in more biased estimators.

\subsection{Varying error rates}

Assumption 2 (2A) suggests to obtain consistent point estimates of coefficients, FNR+ $F P R$ should be smaller than one (or $P P V+N P V$ larger than one). However, if one further cares about hypothesis testing, then even when $F N R+F P R<1$ but is relatively large (say 0.8), the variance estimates may be too large. What amount of prediction error is tolerable if one also cares about hypothesis testing? We repeated Simulations 1 and 2, but let $F N R$ and FPR vary from 0.1 to 0.4 with distance 0.1 .

Figure 8 shows that as $F P R+F P R$ increases (or $P P V+N P V$ decreases), naive regression leads to larger biases. When $F N R+F P R$ is equal to or greater than 0.7 


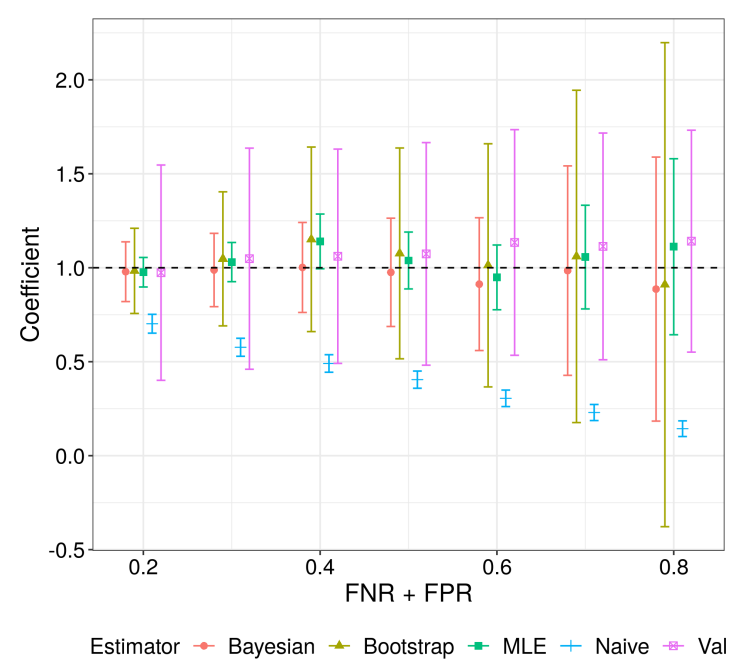

(a) Predicted outcome.

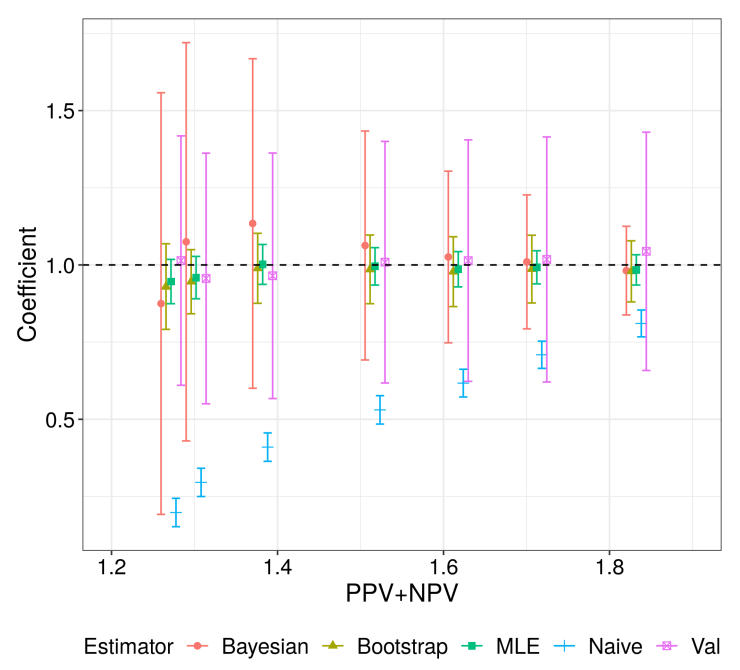

(b) Predicted independent variable.

Figure 8: The effect of ML algorithm performance on coefficient estimates. Increasing $F N R+F P R$ (decreasing $P P V+N P V$ ) indicates more prediction errors. Estimated regression coefficients with $95 \%$ confidence (credible) intervals. The true value of each coefficient is plotted as a dotted horizontal line.

(or $P P V+N P V \leq 1.3$ ), the worst performing estimator for each case-although still giving good point estimates - yields a confidence interval so large that we cannot statistically distinguish it from naive estimates. These numbers can serve as a quick rule of thumb for researchers to use to determine whether their algorithms are accurate enough or need further improvement before using their predictions as variables.

\section{Replications and Results}

All articles in Table 1 directly inserted ML classification into their next-step regressions, which has now been proven to lead to biased (very likely attenuated) effect size estimates. Fortunately, six of the eight studies (Studies $1-6$ ) that used binary classification quantified the performance of their algorithms, which allowed us to revisit them and see if the results still held after considering ML classification errors. 
Interestingly, all six studies are from political science journals.

We applied our estimators to Studies $1-4 .^{33}$ Note that Studies 2 to 4 further aggregated their ML classifications into proportions or counts (of the positive class) by subjects (Study 2), by geographical region (Study 3), or by time (Study 4). We used Equation 2 to obtain the corrected proportions/counts and then used them as outcome variables to estimate the next-step regression. The reestimation details are provided in Appendix O. Note that our reestimations are not intended to refute the original studies; each of the studies tried multiple setups for their regressions and performed extensive robustness checks. Our purpose here is to show how the main results would be different if the authors had incorporated the ML classification error in their main next-step regression models.

Applying our estimators requires Assumptions 1 to 3 to hold. Studies 2 and 3 verified Assumption 1. Studies 1 and 4 did not verify Assumption 1, and we had to proceed assuming that Assumption 1 was true. Assumptions 2 and 3 hold true for Studies $1-4$.

Figure 9 compares the naive estimates with the estimates after applying our estimators. In almost all cases, the new estimates increased effect size, but also increased the standard errors. Studies 1 to 4 can be further classified into two types on the basis of the performance of their ML algorithms. The first group is Study 1 - 3 (Panel a to c). The classifications in these studies are accurate, such that the increase in coefficient size offsets the increase in variances. In the end, applying our statistical correction made the effect size larger while remaining statistically significant.

The second group is Study 4 (Panel d). The ML classification error rates were higher than in Studies 1-3; FNR in Study 4 was as high as 0.5. Additionally, both the outcome and two independent variables were predicted. Consequently, for Study 4, incorporating ML classification errors into next-step regression noticeably widens 
the confidence intervals, such that the regression coefficients are no longer statistically significant. ${ }^{34}$

To summarize, when ML classification errors are taken into consideration slope coefficients tend to be larger in scale, which suggests a stronger effect size. However, the variance of coefficients also increases. If the ML classification is accurate enough, the increase in variance will be small and the effect will still be statistically significant. If the ML classification is not accurate enough, however, the classification errors can increase the variance of the coefficient to such an extent that coefficient estimates will no longer be statistically significant.

\section{Concluding Remarks}

We make three main contributions to the literature. First, we show that treating ML predictions as true measures for independent or dependent variables in regression models will lead to biased (mostly likely attenuated) estimates in regression coefficients. As our detailed literature review has shown, almost all current research in the computational social science community that has used ML algorithms for measurement suffers from such biases, and this can potentially result in estimates which are not only too small in size but also overly confident in rejecting the null hypothesis. Second, we propose new estimators, particular the Bootstrap and Bayesian estimators, to not only obtain consistent coefficients for next-step regression models, but also to properly account for the variances of ML classification error rates themselves when estimating coefficients. Third, our simulations reveal useful properties of our new estimators, such as choice of validation dataset size, range of acceptable ML algorithm performances, what to do when assumptions are violated, and the relative performance of Bootstrap and Bayesian estimators, all of which are decisions 


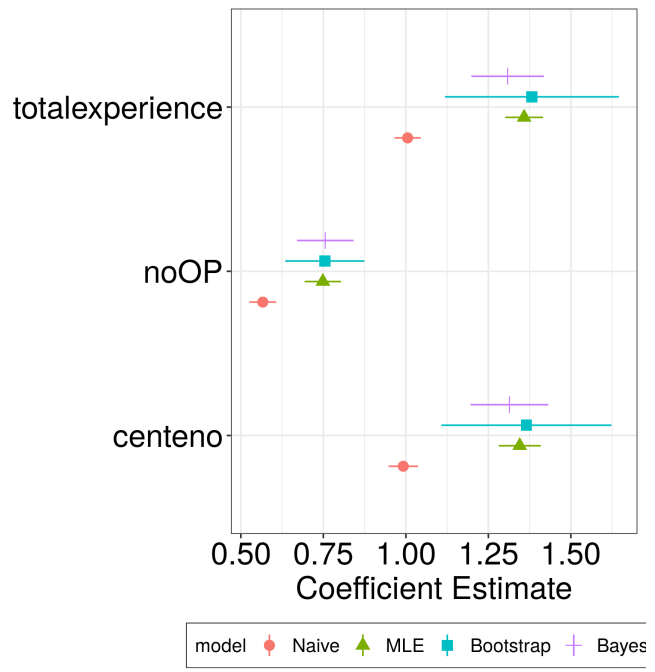

(a) Study 1, Model 1 in Table 2 of Cantu (2019).

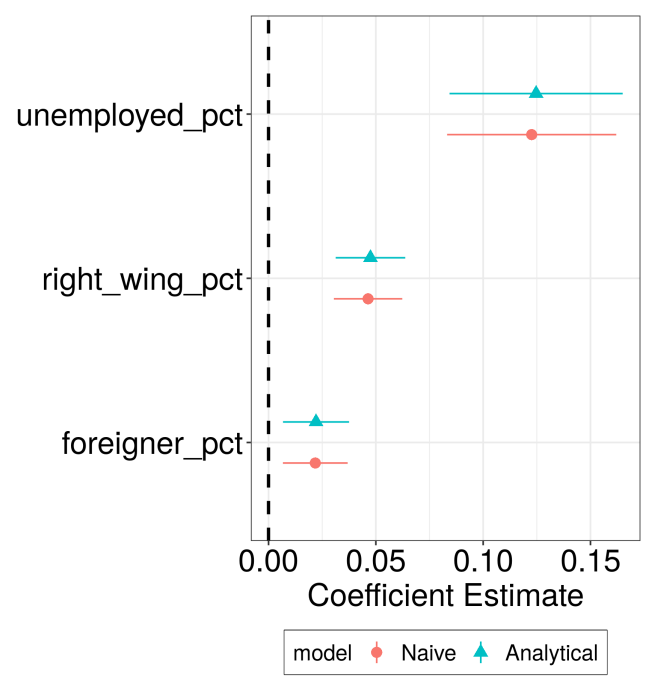

(c) Study 3, Model 1 in Table 6 of Mitts (2019).

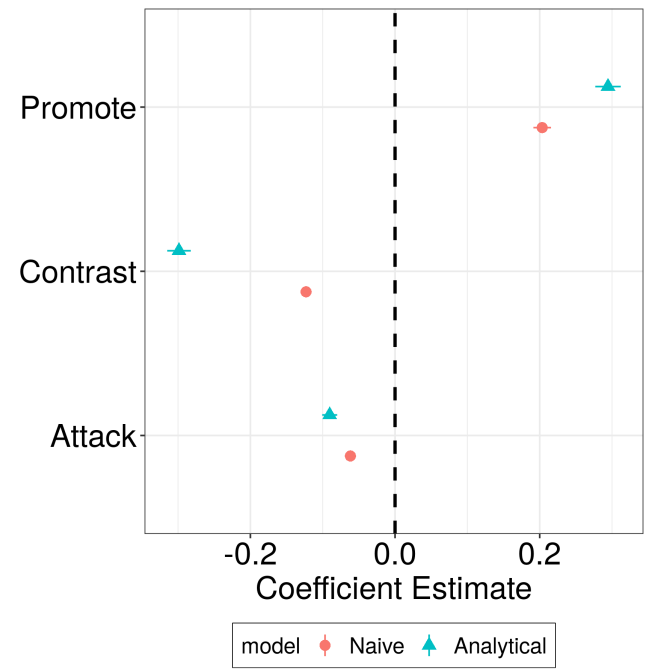

(b) Study 2, Figure 5 of Fowler et al. (2020).

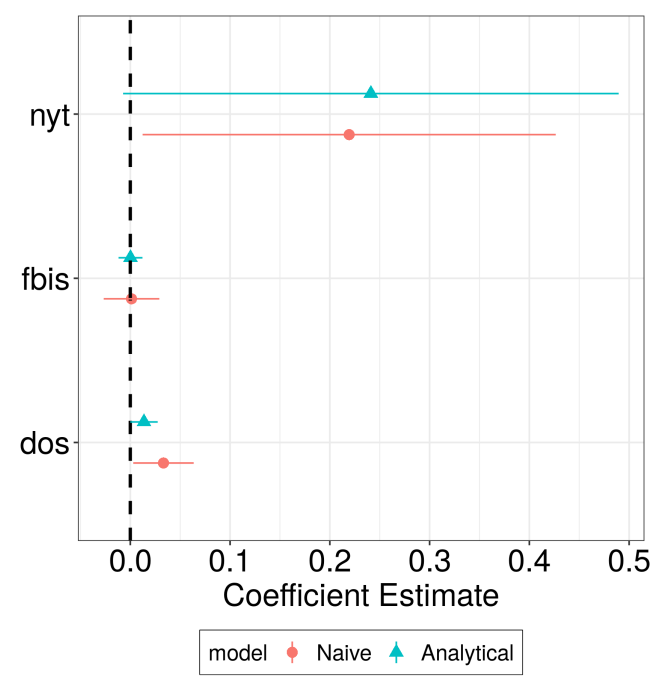

(d) Study 4, Model 1 in Table 2 of Katagiri and Min (2019).

Figure 9: Reestimating real-world studies. All of the studies used ML predictions as the dependent variables. Study 2 also used ML predictions as independent variables. 
which researchers need to make. Finally, we are also developing a $\mathrm{R}$ package to assist researchers who wish to use our estimators.

We provided a checklist for researchers to refer to when using ML predictions in their models:

- Always calculate ML error rates against validation data. This point is easy to satisfy if one has used standard supervised machine learning and trained one's own model, but is often forgotten if one applies dictionary methods or existing models trained by others.

- Check Assumptions 1 and 2. If Assumption 1 is violated, this suggest some selection biases in the creation of the ML classification and could be addressed by improving the research design. If Assumption 2 is on the edge of being broken (e.g. $F N R+F P R>0.7$ ), then one should consider improving the performance of the ML algorithms performances before using the ML predictions in next-step regressions.

- Apply our estimators. Also compare the estimates from using validation data only if one suspects a violation of Assumption 1.

This study has some limitations, which open the door to future research. Possible extensions of the concepts described in this study can be grouped into those improving estimation of classification errors, and those designing statistical models to handle more complex regression types. On improving statistical models, this article does not discuss cases where categorical predictions are used in regression, nor those where multiple independent variables are predicted, nor those where both independent and dependent variables are predicted. It would be straightforward to extend the MLE, Bootstrap, and Bayesian estimators to these situations: one could modify the cor- 
responding likelihood to link observed data probability to true data probability and error rates.

On improving the measurement of ML algorithm prediction errors, we point out three further directions for extending the concepts in this study. First, we have assumed that validation data are measured perfectly without errors, but in reality validation data are often created by multiple coders and may contain coding errors or inconsistencies (Song et al., 2020). Incorporating the influence of intercoder inconsistencies on estimating error rates is a natural next step.

The second extension is to relax Assumption 1 and derive estimators in which the prediction error can be correlated with other variables in the regression. As discussed in Section 6.4, our MLE, Bootstrap and Bayesian estimators are less biased than naive regression, but they are still biased. An relatively easier case is when prediction errors are correlated with observed confounders in the regression (i.e. Figure 6). We can loosen Assumption 1 to be a conditional on observables assumption: that prediction errors are fully explained by observed confounders. Then, one can borrow the socalled regression calibration or simulation extrapolation methods (i.e. SIMEX) that have been well developed in the existing measurement error literature (Carroll et al., 2006, Chap. 4-5). The basic idea is to use validation data to learn a function that models true values $D(Y)$ as a function of predicted values $\tilde{D}(\tilde{Y})$ and other variables that are correlated with prediction errors. We can then use the learned functions to generate calibrated predictions, which will then satisfy Assumption 1 again. A much more challenging case is when one suspects that the prediction errors are correlated with unobserved confounders (i.e. Figure 7). One has to borrow insights from the causal inference literature to address this type of unobserved confounding problem.

The third extension is towards developing better ways to estimate group-specific error rates. As our review of the published research has shown, many scholars will 
use the proportions or count of the positive class as outcomes. It is reasonable to suspect that the error rates of the ML prediction may vary between groups. However, estimating group-specific error rates is challenging because the validation dataset is already small, and further splitting it into subgroups may lead to serious finite-sample biases. In the worst situation (Study 4), each group may not even have a single validation datapoint, let alone to calculate meaningful error rates estimates. When replicating studies that aggregate their binary predictions into counts or proportions in Section 7, we had to assume that the error rates were the same across groups for aggregation. Future research could investigate methods for the estimation of groupspecific error rates by borrowing methods from small area estimation (Molina and Rao, 2010) or hierarchical models (Gelman and Hill, 2006).

Finally, there are many other studies that used predictions from unsupervised machine learning, particularly topic modeling, in next-step regressions, partly because unsupervised methods allowed social scientists to explore their data without extensive data-labeling efforts (Table A.1). Apparently, predictions from unsupervised methods may also contain prediction errors and thus may bias the coefficient estimates. Roberts et al. (2014) realized this problem and designed Structural Topic Models (STM), which incorporates the uncertainty of topic proportions returned from basic topic modeling (Latent Dirichlet Allocation, or LDA) into the estimation of nextstep regression models. This research seeks to address the similar problem raised by Roberts et al. (2014), although it differs in solutions by using an external validation dataset. Influential work that introduced topic modeling into social sciences also recommends that scholars compare their topic modeling results with some external measures of the same theoretical concept by human annotation, a process coined as testing "convergent validity" (Quinn et al., 2010; Grimmer and Stewart, 2013). However, this recommendation is not well followed among applied research. Overall, this 
research cautions against the exercise of treating machine predictions as the truth, and encourages scholars to consider either using our approach or Roberts et al. (2014) to incorporate prediction errors into estimating next-step regressions.

\section{Notes}

${ }^{1}$ A standard image classification ML algorithm.

${ }^{2}$ Because of this requirement, the solutions provided in this article do not work for research that uses predictions from unsupervised methods as variables, because it is hard to define true values when using unsupervised methods. Take topic modeling as an example; some scholars will use the predicted portions of topic models as input in their regression models.

${ }^{3}$ The CCER package can be found at https://anonymous.4open.science/r/CCER-7F1D/

${ }^{4}$ We did not list articles that used machine learning for purposes other than measurement (e.g. forecasting social or political events; estimating heterogeneous treatment effects). There are more published research used ML-predicted variables but did not contain the query terms we used, or were not published in these journals, and they are also not listed in Table 1.

${ }^{5}$ And when mismeasured variable is used as the independent variable, a naive regression leads to attenuation bias of the slope coefficient estimates. See Wooldridge (2015, Chapter 9.4) for a textbook instruction.

${ }^{6}$ The key is that the classical measurement error assumption requires that measurement error $(\widetilde{X}-X)$ is uncorrelated with the truth, or mathematically, $E((\tilde{X}-X) X)=0$. However, binary, categorical, and bounded continuous predictions must produce a negative correlation between the measurement error and the truth $(E((\widetilde{X}-X) X)<0)$. The reason why binary classification must produce non-classical errors is easy to find. When $(\mathrm{X})=0,(\widetilde{X}-X) X$ is automatically 0 ; when $X=1, \widetilde{X}-X$ is either 0 (when the prediction is correct) or -1 (when the prediction is incorrect). Then $(\widetilde{X}-X) X$ must be negative, unless the prediction is perfect. See Black et al. (2000) for discussions of categorical and bounded continuous variable case.

${ }^{7}$ The justification is often to improve statistical efficiency.

${ }^{8}$ Define $\mu=E(\widetilde{X})$ as the mean of the predictions. Then $P P V=\frac{(1-F N R) \mu}{(1-F N R) \mu+F P R(1-\mu)}$. And $N P V=\frac{(1-F P R)(1-\mu)}{(1-F P R)(1-\mu)+F N R \cdot \mu}$ 
${ }^{9}$ Otherwise we could directly use the true values $Y$ in next-step regression, rather than using the predictions.

${ }^{10}$ Hopkins and King (2010) later independently proposed an way to estimate the proportion of positive class of binary classifications. See Appendix F for the proof of equivalence of Hopkins and King (2010) and Equation 2.

${ }^{11}$ Even if outcome is binary, some may prefer linear regression for its mathematical simplicity. See Lowande (2018) for a recent example using linear probability model for binary ML predicted outcomes in a difference-in-differences design.

${ }^{12}$ This equation also holds for other generalized linear models (e.g. probit, Poisson, negative binomials, etc.).

${ }^{13}$ One can always perform colinearity tests between prediction errors and other control variables to see if this extreme situation may occur.

${ }^{14}$ This situation can emerge in two cases. The first case can occur when convenient samples that have been labeled for other purposes are used to train ML algorithms. The convenient training data are not necessarily sampled from the same source of the primary data (Zhang and Pan, 2019). The second case is when researchers intentionally choose nonrepresentative training data to improve algorithm training (e.g. oversampling certain minority or hard-to-predict cases (Miller et al., 2020)).

${ }^{15}$ An alternative non-informative prior is Jeffery's prior $(a=b=0.5)$; it yields similar performance.

${ }^{16}$ Fong and Tyler (2020) first noted $\hat{\beta}^{\text {val }}$ and combined it with an IV estimator.

${ }^{17}$ For instance, Fowler et al. (2020) (Study 2) aggregates their political advertisement data at candidate level. There are 7,298 candidates, but the validation dataset is only of size 2,886, which suggests that there is less than one validation data point in each group (i.e. for each candidate).

${ }^{18}$ Appendix G further shows the equivalence between analytical correction to the IV estimator used in Fong and Tyler (2020), when the ML classification is used as an independent variable and there is no control variable.

${ }^{19}$ If one choose to use linear regression to model this binary outcome, then $p_{i}$ shall be modified to $p_{i}=P\left(Y_{i}=1 \mid D_{i}, W_{i}\right)=\alpha+\beta D_{i}+\gamma W_{i}$; the rest will be the same. Furthermore, because $p_{i}$ is a probability, when estimating MLE we also need to restrict $\alpha+\beta D_{i}+\gamma W_{i}$ to be within $[0,1]$.

${ }^{20}$ The choice of half-Cauchy follows the suggestion by Gelman et al. (2008); Polson and Scott (2012). 
${ }^{21} \mathrm{Or}$, if one use linear probability model, $P(Y=1 \mid D, W)=\alpha+\beta D+\gamma W$ for linear regression. Again, the simulated $P(Y=1 \mid D, W)$ need to be coerced between $[0,1]$.

${ }^{22}$ For Bayesian estimators, the first half of the draws were used as burn-in samples. We found that a smaller number of draws (e.g. 5000) resulted in similar point estimates. For Bootstrap estimators, a much smaller number of draws (1000) was already enough to give a very similar performance to that of 10,000 draws.

${ }^{23}$ Appendix L shows the estimates of the same data using the linear probability model; the findings are similar.

${ }^{24}$ Appendix K describes further diagnostics of Bayesian MCMC sampling.

${ }^{25}$ We define $F N R$ and $F P R$ instead of $P P V$ and $N P V$ because the former is conditioned on the true value, making it easier to simulate the classification from its true value. The formula in Footnote 8 can be used to calculate $P P V$ and $N P V$.

${ }^{26} \mathrm{On}$ the other hand, for ML-predicted independent variables, $P P V$ and $N P V$ are relevant. Bootstrap estimates of $P P V$ and $N P V$ are more precise and thus the Bootstrap estimator also gives more precise coefficient estimates than the Bayesian estimator.

${ }^{27}$ If one sets aside a proportion of training data as validation dataset, then larger validation dataset could also reduce the number of training samples and effectively making the ML algorithms less accurate.

${ }^{28}$ Note this is the correlation between the prediction error and the other variables; the correlation between the predicted variable and other variables is typically larger in scale.

${ }^{29}$ The key is to instruct MLE, Bootstrap, and Bayesian estimators to find parameter values within the $95 \%$ confidence interval set by the regression on validation data. Otherwise the estimates may be inaccurate. Appendix N.1 compares the estimates with and without restricting parameter values.

${ }^{30}$ We used the same setup as the simulations in Figure 6 except that we omitted the independent variable $W$ when running next-step regression.

${ }^{31}$ However, in real-world studies we do not know which direction OVB will be in. Therefore, it would be foolish to rely on chance and assume that the ML classification error and OVB will cancel each other out.

${ }^{32}$ The naive estimate when $\operatorname{cor}(\tilde{Y}-Y, D)$ equals 0.4 was too large $(11.03)$ to be meaningfully plotted here, so we removed it.

${ }^{33}$ We did not replicate Study 5, although $F N R / F P R$ was available, because the author had 
performed further modifications to the raw predictions using a capture-recapture method. We did not replicate Study 6 because it only provides an accuracy measure, not FPR and FNR.

${ }^{34}$ We also estimated another model in that study and found similar results (Appendix O.4)

\section{References}

Aigner, Dennis J. 1973. "Regression with a Binary Independent Variable Subject to Errors of Observation." Journal of Econometrics 1:49-59.

Athey, Susan and Guido W. Imbens. 2019. "Machine Learning Methods That Economists Should Know About." Annual Review of Economics 11:685-725.

Bail, Christopher A., Taylor W. Brown, and Marcus Mann. 2017. "Channeling Hearts and Minds: Advocacy Organizations, Cognitive-Emotional Currents, and Public Conversation." American Sociological Review 82:1188-1213.

Belloni, A., D. Chen, V. Chernozhukov, and C. Hansen. 2012. "Sparse Models and Methods for Optimal Instruments With an Application to Eminent Domain." Econometrica 80:2369-2429.

Bisani, M. and H. Ney. 2004. "Bootstrap Estimates for Confidence Intervals in ASR Performance Evaluation." In 2004 IEEE International Conference on Acoustics, Speech, and Signal Processing, volume 1, pp. I-409.

Black, Dan A., Mark C. Berger, and Frank A. Scott. 2000. "Bounding Parameter Estimates with Nonclassical Measurement Error." Journal of the American Statistical Association 95:739-748.

Bollinger, Christopher R. 1996. "Bounding Mean Regressions When a Binary Regressor Is Mismeasured." Journal of Econometrics 73:387-399. 
Burgess, Robin, Matthew Hansen, Benjamin A. Olken, Peter Potapov, and Stefanie Sieber. 2012. "The Political Economy of Deforestation in the Tropics." The Quarterly Journal of Economics 127:1707-1754.

Cantú, Francisco. 2019. "The Fingerprints of Fraud: Evidence from Mexico's 1988 Presidential Election." American Political Science Review 113:710-726.

Carroll, Raymond J., David Ruppert, Ciprian M. Crainiceanu, and Leonard A. Stefanski. 2006. Measurement Error In Nonlinear Models: A Modern Perspective. Chapman and Hall/CRC.

Cederman, Lars-Erik and Nils B. Weidmann. 2017. "Predicting Armed Conflict: Time to Adjust Our Expectations?" Science 355:474-476.

Chernozhukov, Victor, Denis Chetverikov, Mert Demirer, Esther Duflo, Christian Hansen, Whitney Newey, and James Robins. 2018. "Double/Debiased Machine Learning for Treatment and Structural Parameters." The Econometrics Journal 21:C1-C68.

Crabtree, Charles, Matt Golder, Thomas Gschwend, and Indriđi H. Indriđason. 2020. "It Is Not Only What You Say, It Is Also How You Say It: The Strategic Use of Campaign Sentiment." The Journal of Politics 82:1044-1060.

Flores, René D. 2017. "Do Anti-Immigrant Laws Shape Public Sentiment? A Study of Arizona's SB 1070 Using Twitter Data." American Journal of Sociology 123:333384.

Fong, Christian and Matthew Tyler. 2020. "Machine Learning Predictions as Regression Covariates." Political Analysis pp. 1-18. 
Fowler, Erika Franklin, Michael M. Franz, Gregory J. Martin, Zachary Peskowitz, and Travis N. Ridout. 2020. "Political Advertising Online and Offline." American Political Science Review pp. 1-20.

Frazis, Harley and Mark A. Loewenstein. 2003. "Estimating Linear Regressions with Mismeasured, Possibly Endogenous, Binary Explanatory Variables." Journal of Econometrics 117:151-178.

Gelman, Andrew and Jennifer Hill. 2006. Data Analysis Using Regression and Multilevel/Hierarchical Models. Cambridge ; New York: Cambridge University Press, 1 edition edition.

Gelman, Andrew, Aleks Jakulin, Maria Grazia Pittau, and Yu-Sung Su. 2008. "A Weakly Informative Default Prior Distribution for Logistic and Other Regression Models." The Annals of Applied Statistics 2:1360-1383.

Gohdes, Anita R. 2020. "Repression Technology: Internet Accessibility and State Violence." American Journal of Political Science 64:488-503.

Goldberg, Amir, Sameer B. Srivastava, V. Govind Manian, William Monroe, and Christopher Potts. 2016. "Fitting In or Standing Out? The Tradeoffs of Structural and Cultural Embeddedness." American Sociological Review 81:1190-1222.

Goutte, Cyril and Eric Gaussier. 2005. "A Probabilistic Interpretation of Precision, Recall and F-Score, with Implication for Evaluation." In Advances in Information Retrieval, edited by David E. Losada and Juan M. Fernández-Luna, Lecture Notes in Computer Science, pp. 345-359, Berlin, Heidelberg. Springer.

Grimmer, Justin, Margaret E. Roberts, and Brandon M. Stewart. 2021. "Machine 
Learning for Social Science: An Agnostic Approach." Annual Review of Political Science 24:null.

Grimmer, Justin and Brandon M. Stewart. 2013. "Text as Data: The Promise and Pitfalls of Automatic Content Analysis Methods for Political Texts." Political Analysis 21:267-297.

Hastie, Trevor, Robert Tibshirani, Jerome Friedman, T. Hastie, J. Friedman, and R. Tibshirani. 2009. The Elements of Statistical Learning, volume 2. Springer.

Hausman, J. A., Jason Abrevaya, and F. M. Scott-Morton. 1998. "Misclassification of the Dependent Variable in a Discrete-Response Setting." Journal of Econometrics $87: 239-269$.

Hopkins, Daniel J. and Gary King. 2010. "A Method of Automated Nonparametric Content Analysis for Social Science." American Journal of Political Science 54:229247.

Imai, Kosuke and Teppei Yamamoto. 2010. "Causal Inference with Differential Measurement Error: Nonparametric Identification and Sensitivity Analysis." American Journal of Political Science 54:543-560.

Katagiri, Azusa and Eric Min. 2019. "The Credibility of Public and Private Signals: A Document-Based Approach." American Political Science Review 113:156-172.

Lee, Lung-Fei and Jungsywan H. Sepanski. 1995. "Estimation of Linear and Nonlinear Errors-in-Variables Models Using Validation Data." Journal of the American Statistical Association 90:130-140.

Lewbel, Arthur. 2000. "Identification of the Binary Choice Model with Misclassification." Econometric Theory 16:603-609. 
Lowande, Kenneth. 2018. "Who Polices the Administrative State?" American Political Science Review 112:874-890.

Meyer, Bruce D. and Nikolas Mittag. 2017. "Misclassification in Binary Choice Models." Journal of Econometrics 200:295-311.

Miller, Blake, Fridolin Linder, and Walter R. Mebane Jr. 2020. "Active Learning Approaches for Labeling Text: Review and Assessment of the Performance of Active Learning Approaches." Political Analysis .

Mitts, Tamar. 2019. "From Isolation to Radicalization: Anti-Muslim Hostility and Support for ISIS in the West." American Political Science Review 113:173-194.

Molina, Isabel and J. N. K. Rao. 2010. "Small Area Estimation of Poverty Indicators." Canadian Journal of Statistics 38:369-385.

Molina, Mario and Filiz Garip. 2019. "Machine Learning for Sociology." Annual Review of Sociology 45:27-45.

Paxton, Pamela, Kristopher Velasco, and Robert W. Ressler. 2020. "Does Use of Emotion Increase Donations and Volunteers for Nonprofits?" American Sociological Review 85:1051-1083.

Polson, Nicholas G. and James G. Scott. 2012. "On the Half-Cauchy Prior for a Global Scale Parameter." Bayesian Analysis 7:887-902.

Quinn, Kevin M., Burt L. Monroe, Michael Colaresi, Michael H. Crespin, and Dragomir R. Radev. 2010. "How to Analyze Political Attention with Minimal Assumptions and Costs." American Journal of Political Science 54:209-228.

Roberts, Margaret E., Brandon M. Stewart, Dustin Tingley, Christopher Lucas, Jetson Leder-Luis, Shana Kushner Gadarian, Bethany Albertson, and David G. Rand. 
2014. "Structural Topic Models for Open-Ended Survey Responses." American Journal of Political Science 58:1064-1082.

Sakai, Tetsuya. 2006. "Evaluating Evaluation Metrics Based on the Bootstrap." In Proceedings of the 29th Annual International ACM SIGIR Conference on Research and Development in Information Retrieval, SIGIR '06, pp. 525-532, New York, NY, USA. Association for Computing Machinery.

Salganik, Matthew. 2019. Bit by Bit: Social Research in the Digital Age. Princeton University Press.

Schennach, Susanne M. 2016. "Recent Advances in the Measurement Error Literature." Annual Review of Economics 8:341-377.

Shor, Eran, Arnout van de Rijt, Alex Miltsov, Vivek Kulkarni, and Steven Skiena. 2015. "A Paper Ceiling: Explaining the Persistent Underrepresentation of Women in Printed News." American Sociological Review 80:960-984.

Song, Hyunjin, Petro Tolochko, Jakob-Moritz Eberl, Olga Eisele, Esther Greussing, Tobias Heidenreich, Fabienne Lind, Sebastian Galyga, and Hajo G. Boomgaarden. 2020. "In Validations We Trust? The Impact of Imperfect Human Annotations as a Gold Standard on the Quality of Validation of Automated Content Analysis." Political Communication 37:550-572.

Wager, Stefan and Susan Athey. 2018. "Estimation and Inference of Heterogeneous Treatment Effects Using Random Forests." Journal of the American Statistical Association 113:1228-1242.

Wooldridge, Jeffrey M. 2015. Introductory Econometrics: A Modern Approach. Boston, MA: Cengage Learning, 6 edition edition. 
Zhang, Han and Jennifer Pan. 2019. "CASM: A Deep-Learning Approach for Identifying Collective Action Events with Text and Image Data from Social Media." Sociological Methodology 49:1-57. 


\section{Appendix A Studies using unsupervised machine learn- ing algorithms in top journals}

See Table A.1. All we can found uses topic modeling.

\section{Appendix B Using the predicted probability in re- gression}

This simulation shows that using the predicted probabilities in next-step regression still leads to biases in the coefficient estimates, even if the true values of the predictions are also continuous.

Predicted probability as outcome variable For the case of using the predicted probability as the outcome variable, the data generating process (DGP) is as follows:

- $D, W \sim N(0,1)$

- $Y^{*}=\operatorname{logit}^{-1}(\alpha+\beta * D+\gamma * W) ; Y^{*}$ is true data probability; $\epsilon \sim N(0,1)$ is an error term; $\alpha, \beta, \gamma$ are coefficients for intercept, $D$ and $W$, respectively.

- $Y_{p}^{*}=Y^{*}+N(0,0.2) ; Y_{p}^{*}$ is predicted data probability.

We sampled 50 sampled datasets to smooth the estimates. For each sampled dataset, 9,100 data points from the above DGP (100 for validation data and the rest 9,000 for the primary data). We set $\alpha=0.5, \beta=1, \gamma=1$ in the simulation.

It is easy to see that the the above DGP results in a mean prediction error of $0\left(E\left(Y_{p}^{*}-Y^{*}\right)=0\right)$. Yet, as Black et al. (2000) suggests, the correlation between measurement error and truth will be negative, since predictions are continuous 


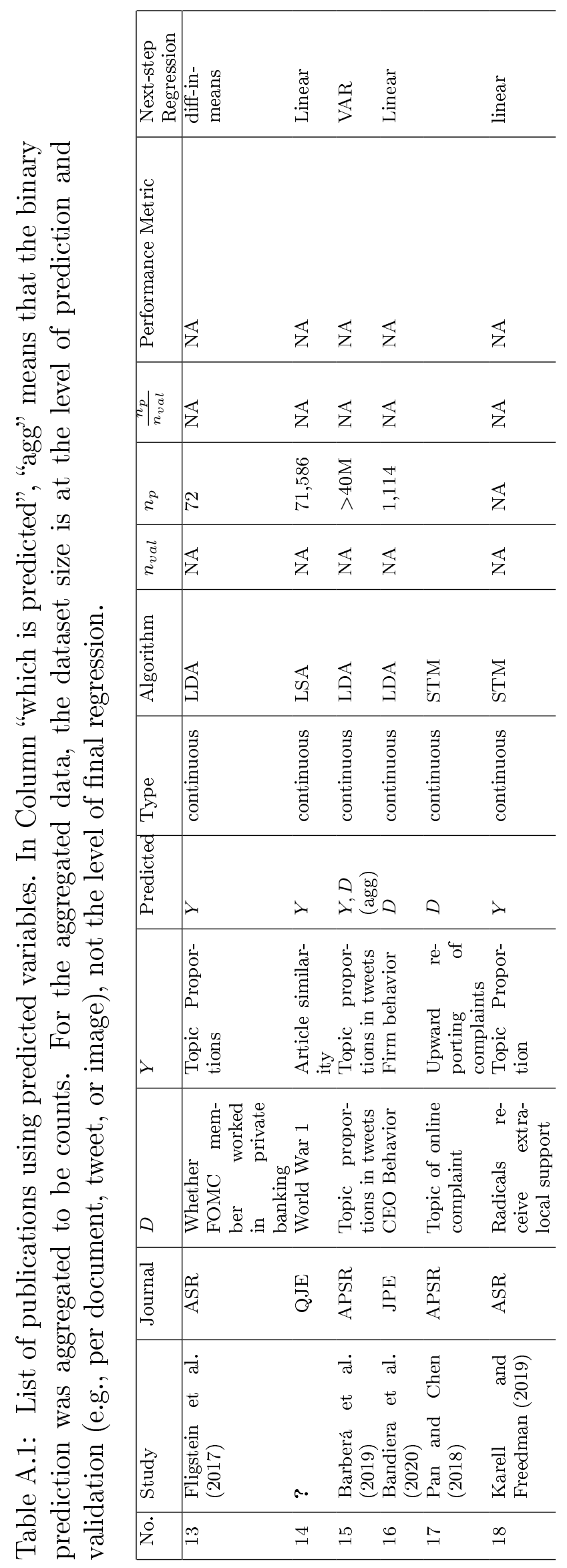


predicted probabilities. Indeed, the above DGP produces a negative correlation: $\left.\operatorname{cor}\left(Y_{p}^{*}-Y^{*}, Y^{*}\right)=-0.16\right)$. It means that we have nonclassical measurement error. This nonclassical measurement error will result in biased coefficient estimates, even when the continuous variable is used as the dependent variables. Figure B.1a shows that using predicted probability as the outcome variable creates serious biases in the coefficient estimates.

Predicted probability as independent variable For the case of using the predicted probability as the independent variable, the DGP is as follows:

- $D^{*} \sim \operatorname{logit}^{-1}(N(0,1))$ is true probability; $W \sim N(0,1)$ is an additional control variable independent of $D ; \alpha, \beta, \gamma$ are coefficients for intercept, $D$ and $W$, respectively.

- $D_{p}^{*}=D^{*}+N(0,0.2) ; D_{p}^{*}$ is a predicted probability. We capped it between $[0,1]$ to make it a probability.

- $Y=0.5+D^{*}+W+\epsilon ; \epsilon \sim N(0,1)$ is an error term.

The results were averaged over 50 sampled datasets to smooth the estimates. Each sampled dataset contains 9,100 data points from the above DGP (100 for validation data and the rest 9,000 for the primary data). We set $\alpha=0.5, \beta=1, \gamma=1$ in the simulation. These parameter choices lead to a negative correlation coefficient between the measurement error and the truth is negative $\left(\operatorname{cor}\left(Y_{p}^{*}-Y^{*}, Y^{*}\right)=-0.12\right)$ in our simulation), again leading to nonclassical measurement error. Figure B.1b shows that using the predicted probability as an independent variable creates serious biases in the coefficient estimates. 


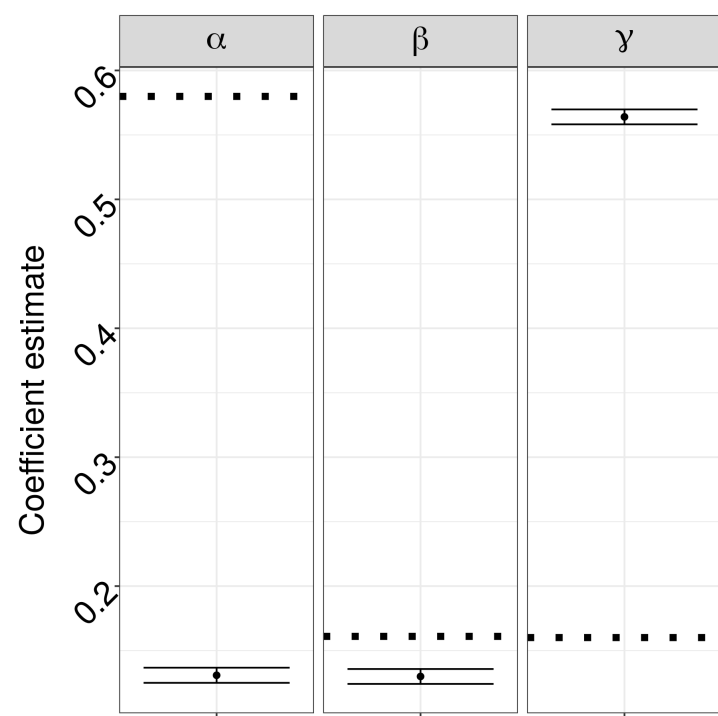

(a) Predicted probability as outcome.

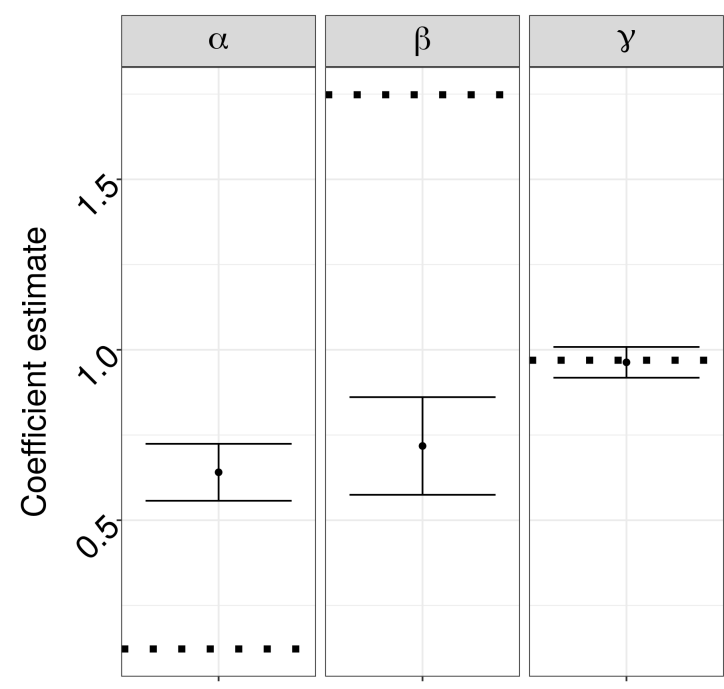

(b) Predicted probability as independent variable.

Figure B.1: Fitting next-step regression using predicted probability as the dependent variables. True values of the coefficients are plotted as dotted lines in each figure.

\section{Appendix C Visualize Scale of Attenuation}

As we discussed in the main text, when the outcome is predicted, the naive slope coefficient is attenuated by a factor of $1-F N R-F P R$. This attenuation factor is known as Youden's Index. Youden's Index can be visualized on an ROC curve (receiver operating characteristic curve), which is widely used to visualize prediction performances (Figure C.1). Given a set of predictions and their true values, the ROC curve plots $F P R$ (as the x-axis) against $1-F N R$ (as the y-axis; also known as the true positive rate). An algorithm that makes random guesses would produce a $45^{\circ} \mathrm{ROC}$ curve; a perfect algorithm's ROC curve would consist of two vertical lines between $(0,0),(0,1)$, and $(0,1),(1,1)$. The scale of attenuation, or the Youden's index, is the vertical distance between a point on the ROC curve and the random guess line. The larger the vertical distance, the better the prediction performance, and the smaller the attenuation. 


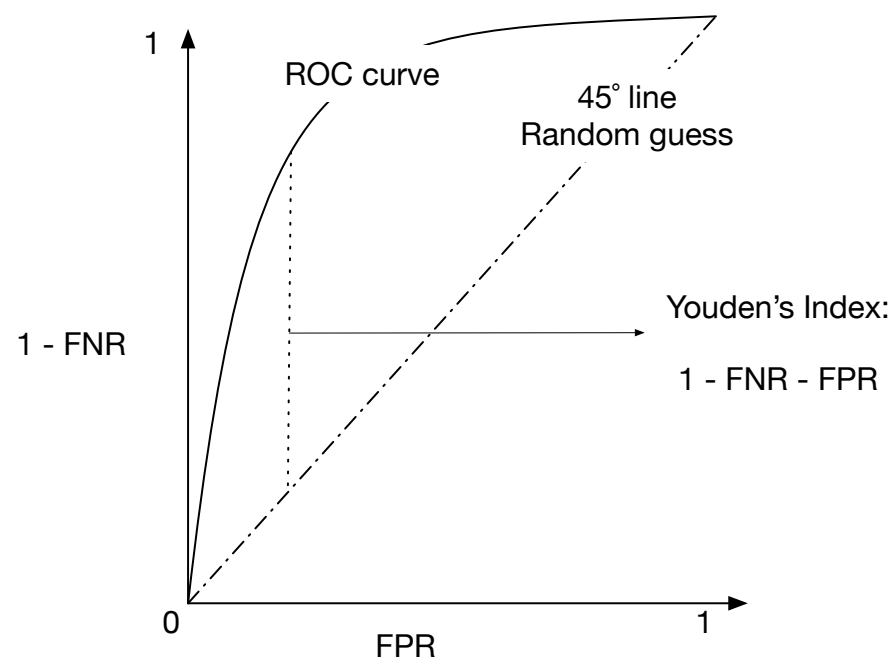

Figure C.1: Visualization of the scale of attenuation $1-F N R-F P R$ on an ROC curve. The scale of attenuation is the vertical distance between a point on the ROC curve and the $45^{\circ}$ random guess line.

\section{Appendix D Estimating ML classification rates: de- tails}

K-fold cross-validation In practice, creating training data is costly. $K$-fold crossvalidation is often recommended when the size of the validation dataset is small. See Hastie et al. (2009, Chap. 7.10) for a detailed discussion of cross-validation. In the following, we describe the general procedures for using cross-validation to calculate the validation data size.

$K$-fold cross-validation splits the data into $K$ parts of equal size. For the $k$ th part, one should train her ML algorithm using the rest $K-1$ parts, and estimates $F P R_{k}, F P R_{k}$ or $P P V_{k}, N P V_{k}$. She should do this process for $k=1,2, \cdots, K$, which will result in $K F P R_{k}$ estimates. The final $\widehat{F P R}=\frac{1}{K} \sum_{i=1}^{K} F P R_{k}$. Other error/performance rates can be obtained similarly.

When $K=N(N$ is the size of the training data), $\widehat{F P R}$ is approximately unbiased 
for true FPR. This is known as the leave-out-one cross-validation. However, this choice will lead to high variance estimates in $F P R$ due to the bias-variance trade-off. Moreover, one needs to train the algorithm $N$ times, which can be unbearably slow.

In practice, many choose to set $K$ to 5 or 10 . This choice will have higher biases in estimating $F P R$ than leave-out-one cross-validation, but result in lower variances in estimation. It is also much faster to calculate. Almost all of the research in Table 1 used 5- or 10-fold cross-validation.

Note that if one uses $k$-fold cross-validation, because each time only one fold of the training data is held out for validation purpose, the effective sample size of validation dataset is $N / k$. For example, Fowler et al. (2020)'s training data size is 14,430 , but because they used 5-fold cross-validation to calculate $F N R / F P R$, the effective size of the validation dataset should be roughly $14,430 / 5=2,886$.

\section{Appendix E Analytical correction for regression co- efficients and standard errors}

\section{E.1 Predicted outcome}

When the ML classification is used as the outcome in linear regression, and there is

no control variable $W$ in the regression, $\hat{\beta}^{\text {analytical }}$ is a consistent estimator for $\beta$ and $\hat{\alpha}^{\text {analytical }}$ is a consistent estimator for intercept $\alpha$ :

$$
\begin{aligned}
\hat{\beta}^{\text {analytical }} & =\frac{\hat{\beta}^{\text {naive }}}{1-\widehat{F N R}-\widehat{F P R}} \\
\hat{\alpha}^{\text {analytical }} & =\frac{\hat{\alpha}^{\text {naive }}-\widehat{F P R}}{1-\widehat{F N R}-\widehat{F P R}}
\end{aligned}
$$


And the variance of the regression coefficient can also be obtained using the Delta method:

$$
\begin{aligned}
\widehat{\operatorname{Var}}\left(\hat{\beta}^{\text {analytical }}\right) & =\widehat{\operatorname{Var}}\left(\frac{\hat{\beta}^{\text {naive }}}{1-\widehat{F N R}-\widehat{F P R}}\right) \\
& =\frac{1}{(1-\widehat{F N R}-\widehat{F P R})^{2}} \widehat{\operatorname{Var}}\left(\hat{\beta}^{\text {naive }}\right)
\end{aligned}
$$

\section{E.2 Predicted independent variable}

When ML classification is used as an independent variable, and no additional control variables $W$ exists in the linear regression, $\hat{\beta}^{\text {analytical }}$ is a consistent estimator for $\beta$ :

$$
\begin{gathered}
\hat{\beta}^{\text {analytical }}=\frac{\hat{\beta}^{\text {naive }}}{\widehat{P P V}+\widehat{N P V}-1} . \\
\hat{\alpha}^{\text {analytical }}=\hat{E}(Y)-\hat{\beta}^{\text {analytical }} \cdot \hat{E}(D),
\end{gathered}
$$

where $\hat{E}(Y)$ is the expectation of the result, which can be estimated as the sample mean of the true result in the validation data.

The variance of $\hat{\beta}^{\text {analytical }}$ can be easily obtained using the Delta method:

$$
\begin{aligned}
\widehat{\operatorname{Var}}\left(\hat{\beta}^{\text {analytical }}\right) & =\widehat{\operatorname{Var}}\left(\frac{\hat{\beta}^{\text {naive }}}{\widehat{P P V}+\widehat{N P V}-1}\right) \\
& =\frac{1}{(\widehat{P P V}+\widehat{N P V}-1)^{2}} \widehat{\operatorname{Var}}\left(\hat{\beta}^{\text {naive }}\right),
\end{aligned}
$$




\section{E.3 Statistical efficiency between MLE estimators and estima- tor based on validation data only}

We first derive the standard error comparison for the predicted independent variable case. Following the well-known properties of linear regression, the standard error of

$\hat{\beta}^{\text {val }}$ converges to true value at the speed of $\frac{1}{n_{\text {val }}^{-1 / 2}}$. The standard error of $\hat{\beta}^{\text {analytical }}$ converges to true value at the speed $\frac{1}{n^{-1 / 2}(P P V+N P V-1)}$. Take the ratio of two convergence rates, we have:

$$
\begin{aligned}
\frac{\widehat{\sigma}^{\text {analytical }}}{\widehat{\sigma}_{\text {val }}} & =\frac{\frac{1}{n^{-1 / 2}(\widehat{P P V}+\widehat{N P V}-1)}}{\frac{1}{n_{\text {val }}^{-1 / 2}}} \\
& =\sqrt{\frac{n_{\text {val }}}{n}} \frac{1}{\widehat{P P V}+\widehat{N P V}-1}
\end{aligned}
$$

From the above formula, it is easy to see that if the following inequality holds, the variance estimate after applying the analytical correction will be smaller than the variance estimate based on validation data only.

$\sqrt{\frac{n_{p}}{n_{\text {val }}}}(P P V+N P V-1)>1$, ML classification as independent variable,

where $n_{p}$ and $n_{v a l}$ are size of primary and validation data.

Similarly, we can derive that if the following inequality holds, the variance estimate after applying the analytical correction will be smaller than the variance estimate based on validation data only: 


$$
\sqrt{\frac{n_{p}}{n_{\text {val }}}}(1-F P R-F N R)>1, \text { ML classification as outcome }
$$

\section{Appendix F Equivalence between Hopkins and King (2010) and the proposed analytical cor- rection in this article}

Hopkins and King (2010) provide an estimator for the proportion of positive documents in a corpus (Equation 2 in their article):

$$
P(Y=1)=\frac{P(\tilde{Y}=1)-(1-\text { specificity })}{\text { sensitivity }-(1-\text { specificity })}
$$

It is well understood that in linear regression with no regressor, intercept $\alpha$ becomes the mean of outcome $E(Y)$; For binary outcome $Y, E(Y)$ further equals the proportion of $Y$ that equals one, $P(Y=1)$. In addition, note that $F P R=$ 1 - specificity, and $F N R=1$ - sensitivity. Therefore, Equation ?? in the present research and Equation 2 in Hopkins and King (2010) is equivalent. Hopkins and King (2010) is a special case of current research because this research also discusses how to obtain slope coefficient estimates beyond the intercept. 


\section{Appendix G Equivalence between IV estimator and the proposed analytical correction in this article}

The IV estimators proposed in Lee and Sepanski (1995) and further adapted by Fong and Tyler (2020) rely on the following (modified) two-stage least squares procedures listed below ${ }^{35}$ :

1. Regress $D$ on $\widetilde{D}$ based on the validation data $(D=\eta+\gamma \widetilde{D}+\delta)$. Then use the estimated $\hat{\eta}, \hat{\gamma}$ to calculated predicted values for $\widetilde{D}$ in the primary data, i.e. $\hat{D}=\hat{\eta}+\hat{\gamma} \widetilde{D}+\epsilon$.

2. Regress $Y$ on $\hat{D}$ for $\hat{D}$ in the primary data. The obtained regression coefficient $\hat{\beta}^{I V}$ is a consistent estimator for $\beta$.

This two-stage least squares estimator is equivalent to Equation 12. The connection is made through rewriting the above modified two-stage least squares procedure as a standard Wald estimator for instrumental variable:

$$
\begin{aligned}
\hat{\beta}^{I V} & =\frac{E(Y \mid \widetilde{D}=1)-E(Y \mid \widetilde{D}=0)}{E(D \mid \widetilde{D}=1)-E(D \mid \widetilde{D}=0)} \\
& =\frac{\hat{\beta}^{\text {naive }}}{P(D=1 \mid \widetilde{D}=1)-P(D=1 \mid \widetilde{D}=0)} \\
& =\frac{\hat{\beta}^{\text {naive }}}{P P V+N P V-1} \\
& =\hat{\beta}^{\text {analytical }}
\end{aligned}
$$




\section{Appendix H MLE for predicted outcomes}

The conditional probability based on the predicted value can be expressed as a function of conditional probability based on the true values:

$$
\begin{aligned}
P(\widetilde{Y}=1 \mid D, W) & =P(\widetilde{Y}=1 \mid D, Y=1) P(Y=1 \mid D, W)+ \\
& P(\widetilde{Y}=1 \mid D, Y=0) P(Y=0 \mid D, W),(\text { Bayes rule }) \\
& =P(\widetilde{Y}=1 \mid Y=1) P(Y=1 \mid D, W)+ \\
& P(\widetilde{Y}=1 \mid Y=0) P(Y=0 \mid D, W),(\text { Assumption } 1) \\
& =(1-F P R) P(Y=1 \mid D, W)+F P R(1-P(Y=1 \mid D, W)),(\text { Definition }) \\
& =(1-F P R-F N R) P(Y=1 \mid D, W)+F P R
\end{aligned}
$$

And,

$$
\begin{aligned}
P(\tilde{Y}=0 \mid D, W) & =1-P(\tilde{Y}=1 \mid D, W) \\
& =1-F P R-(1-F N R-F P R) P(Y=1 \mid D, W)
\end{aligned}
$$

And the observed data likelihood can be expressed as a function of the conditional probability based on true data: 


$$
\begin{aligned}
\log L= & \sum_{i=1}^{N} \log P\left(\widetilde{Y}_{i} \mid D_{i}, W\right) \\
= & \sum_{i=1}^{N} \widetilde{Y}_{i} \log P\left(\widetilde{Y}_{i}=1 \mid D_{i}, W_{i}\right)+\left(1-\widetilde{Y}_{i}\right) \log P\left(\widetilde{Y}_{i}=0 \mid D_{i}, W_{i}\right) \\
= & \sum_{i=1}^{N} \widetilde{Y}_{i} \log ((1-F P R-F N R) P(Y=1 \mid D, W)+F P R) \\
& \quad+\left(1-\widetilde{Y}_{i}\right) \log (1-F P R-(1-F N R-F P R) P(Y=1 \mid D, W))
\end{aligned}
$$

If a linear probability model is used, the conditional probability based on true data is just the linear sum of coefficients and predictors, capped between $[0,1]$ :

$$
P\left(Y_{i} \mid D_{i}, W_{i}\right)= \begin{cases}1 & \alpha+\beta D_{i}+\gamma W_{i}>1 \\ \alpha+\beta D_{i}+\gamma W_{i} & 0<\alpha+\beta D_{i}+\gamma W_{i}<1 \\ 0.0001 & \alpha+\beta D_{i}+\gamma W_{i} \leq 0\end{cases}
$$

We used 0.0001 instead of 0 to make $\log ()$ meaningful. If logistic regression is used, the conditional probability based on true data $(P(Y=1 \mid D, W)$ is linked to the explanatory variables through the inverse logit function (also known as sigmoid function):

$$
P\left(Y_{i}=1 \mid D_{i}, W_{i}\right)=\operatorname{logit}^{-1}\left(\alpha+\beta D_{i}+\gamma W_{i}\right)
$$

Substituting the corresponding expressions of $P(Y=1 \mid D, W)$ into Equation 23, we obtain MLE for next-step regression with classification of ML as a result. 


\section{Appendix I MLE for predicted independent variables}

Observed data log-likelihood is ( $W$ is additional control variable):

$$
\begin{aligned}
\log L & =\sum_{i=1}^{N} \log P\left(Y_{i} \mid \widetilde{D_{i}}, W\right) \\
& =\sum_{i=1}^{N} \widetilde{D_{i}} \log P\left(Y_{i} \mid \widetilde{D_{i}}=1, W\right)+\left(1-\widetilde{D_{i}}\right) \log P\left(Y_{i} \mid \widetilde{D_{i}}=0, W\right)
\end{aligned}
$$

Notice that we can express the conditional probability based on predicted data as a function of the conditional probability based on true $D$,

$$
\begin{aligned}
P(Y \mid \widetilde{D}=1, W) & =P(Y \mid \widetilde{D}=1, D=1, W) P(D=1 \mid \widetilde{D}=1)+P(Y \mid \widetilde{D}=1, D=0, W) P(D=0 \mid \widetilde{D}=1) \\
& =P(Y \mid D=1, W) P P V+P(Y \mid D=0, W)(1-P P V)
\end{aligned}
$$

And,

$$
\begin{aligned}
P(Y \mid \widetilde{D}=0, W) & =P(Y \mid \widetilde{D}=0, D=1, W) P(D=1 \mid \widetilde{D}=0)+P(Y \mid \widetilde{D}=0, D=0) P(D=0 \mid \widetilde{D}=0) \\
& =P(Y \mid D=1, W)(1-N P V)+P(Y \mid D=0, W) N P V
\end{aligned}
$$

In linear regression, the conditional probability based on true $D$ links with the 
true regression coefficients through a normal density function:

$$
P\left(Y_{i} \mid D_{i}, W_{i}\right)=\frac{1}{\sqrt{2 \pi} \sigma^{2}} e^{-\frac{\left(y_{i}-\alpha-\beta D_{i}-\gamma W_{i}\right)^{2}}{2 \sigma^{2}}}
$$

Further denote $p_{i}^{1}=P\left(Y_{i} \mid D_{i}=1, W_{i}\right)$ and $p_{i}^{0}=P\left(Y_{i} \mid D_{i}=0, W_{i}\right)$. Substituting the expressions into Equation 24, we obtain:

$$
\begin{aligned}
\log L= & \sum_{i=1}^{N} \widetilde{D_{i}} \log \left(P\left(Y_{i} \mid D_{i}=1, W_{i}\right) P P V+P\left(Y \mid D_{i}=0, W_{i}\right)(1-P P V)\right)+ \\
& \left(1-\widetilde{D_{i}}\right) \log \left(P\left(Y_{i} \mid D_{i}=1, W_{i}\right)(1-N P V)+P\left(Y_{i} \mid D_{i}=0, W_{i}\right) N P V\right) \\
= & \sum_{i=1}^{n} \tilde{D}_{i} \ln \left[P P V p_{i}^{1}+(1-P P V) p_{i}^{0}\right]+\left(1-\tilde{D}_{i}\right) \ln \left[(1-N P V) p_{i}^{1}+N P V p_{i}^{0}\right]
\end{aligned}
$$

Hence, we obtain MLE for the next-step regression with ML classification as an independent variable.

$$
\hat{\alpha}^{M L E}, \hat{\beta}^{M L E}, \hat{\gamma}^{M L E}=\underset{\alpha, \beta, \gamma}{\arg \max } \log L
$$

where $\log$-likelihood $\log L$ for predicted data is

$\log \mathrm{L}=\sum_{i=1}^{n} \tilde{D}_{i} \ln \left[P P V p_{i}^{1}+(1-P P V) p_{i}^{0}\right]+\left(1-\tilde{D}_{i}\right) \ln \left[(1-N P V) p_{i}^{1}+N P V p_{i}^{0}\right] .(30)$

- $p_{i}^{1}=P\left(Y_{i} \mid D_{i}=1, W_{i}\right)$ and $p_{i}^{0}=P\left(Y_{i} \mid D_{i}=0, W_{i}\right)$

- $P\left(Y_{i} \mid D_{i}, W_{i}\right)=\frac{1}{\sqrt{2 \pi}} e^{-\frac{\left(y_{i}-\alpha-\beta D_{i}-\gamma W_{i}\right)^{2}}{2}}$ is a standard normal density function. 


\section{Appendix J Bayesian estimator for predicted inde- pendent variable in linear regression}

- $P P V, N P V \sim \operatorname{Beta}(1,1)$;

- $T P \sim \operatorname{binomial}(P P V, T P+F P)$;

- $T N \sim \operatorname{binomial}(N P V, T N+F N)$;

- $1 / \sigma^{2} \sim$ half - Cauchy $(0,2.5)$;

- $\alpha, \beta, \gamma \sim$ Truncated $-\operatorname{Normal}\left(0, \sigma^{2} ; v_{\text {lower }}, v_{\text {upper }}\right)$; The use of truncated normal distribution restrict parameter estimates to be within $v_{\text {lower }}, v_{\text {upper }}$, which is the lower and upper bound of the $95 \%$ confidence intervals from coefficient estimates of validation dataset regression.

- $E(Y \mid D, W)=\alpha+\beta D+\gamma W$

- $E(Y \mid \widetilde{D}, W)=D \cdot[E(Y \mid D=1, W) P P V+E(Y \mid D=0, W)(1-P P V)]+(1-$ $D) \cdot[E(Y \mid D=1, W)(1-N P V)+E(Y \mid D=0, W) N P V]$; this line can be obtained based on Equation 23 and 24.

- $\left.Y \sim N\left(E(Y \mid \widetilde{D}, W), \sigma^{2}\right)\right)$.

\section{Appendix K Bayesian estimator diagnostics}

Below is the output of the JAGS software for Simulation 1 (one simulation out of 50). of particular interest is Rhat $(\hat{R})$, which is also known as Gelman-Rubin convergence diagnostic. $\hat{R}<1.1$ is regarded as evidence that Bayesian MCMC sampling has converged. 


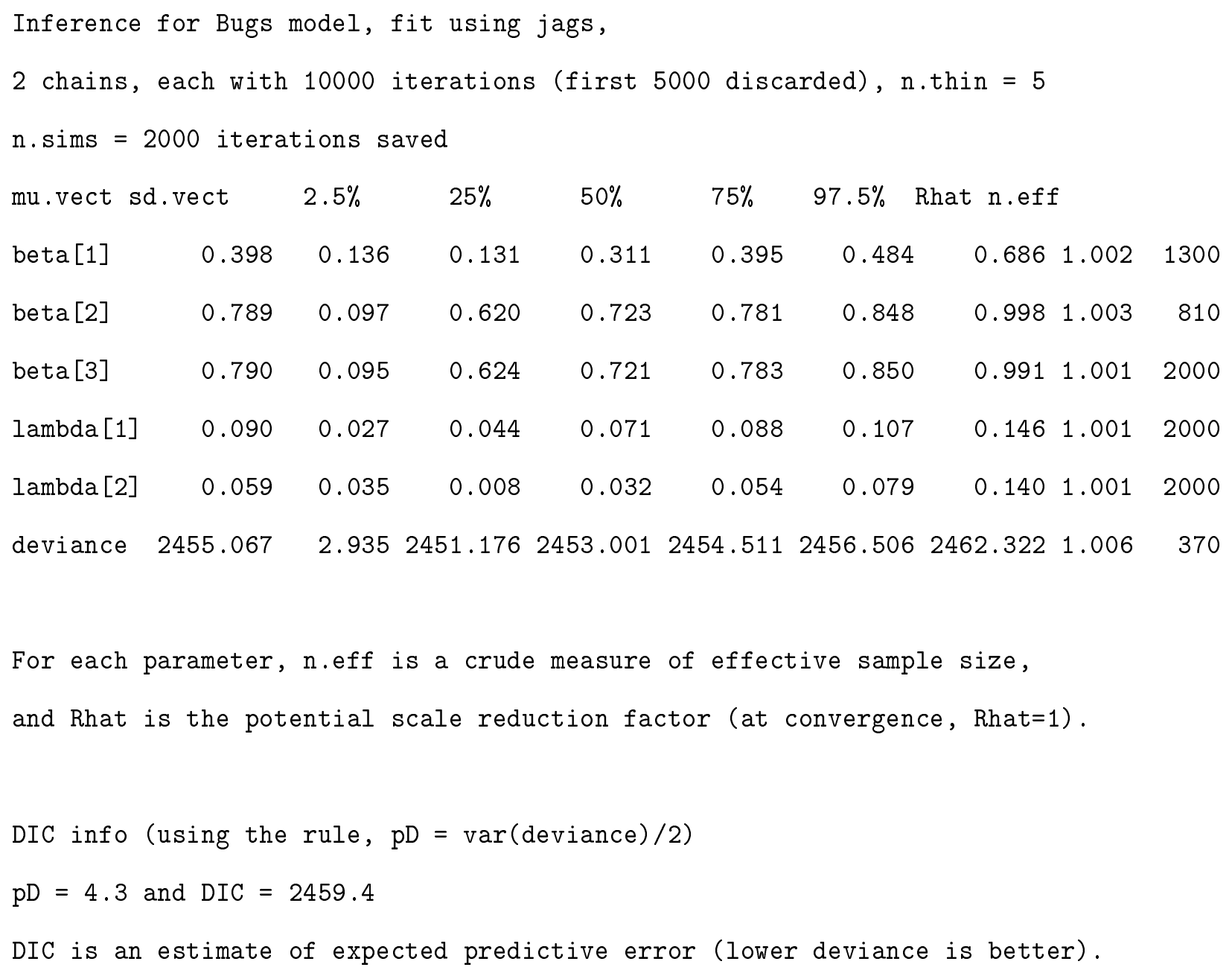

The MCMC sampling trace plot from the above simulation is shown in Figure K.1. Other MCMC traceplots are similar and can be provided upon request. 

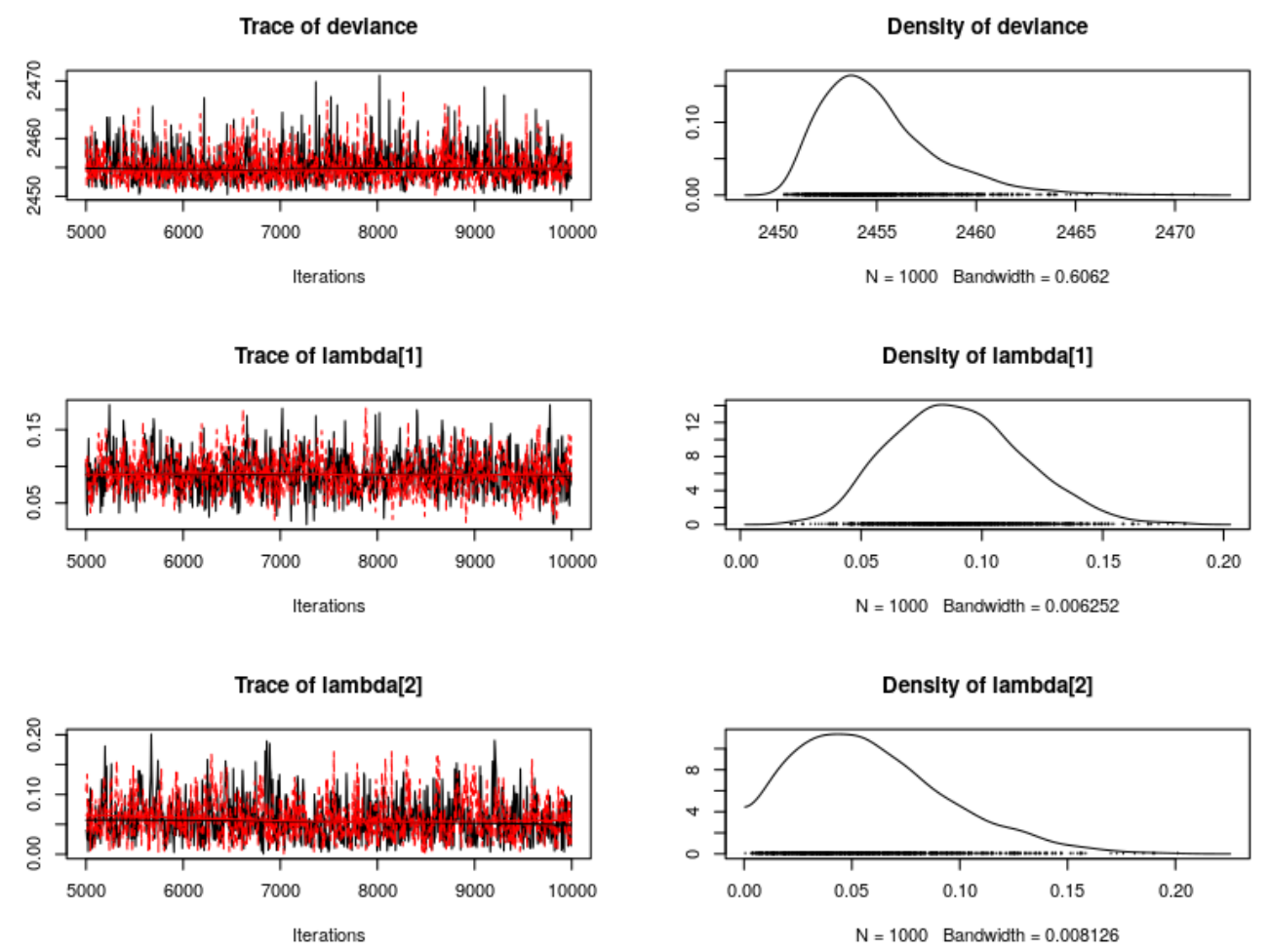

Figure K.1: Trace plot of MCMC sampling for logistic regression. lambda[1] and lambda[2] are FNR and FPR, respectively. beta[1] to beta[3] are $\alpha, \beta, \gamma$ respectively. 


\section{Appendix L Simulation: ML classification as out- come variable in linear probability model}

Figure L.1 plots the coefficient estimates using linear regression, using the same DGP in Simulation $1 .^{36}$ The MLE, Bayesian and Bootstrap estimators again recover the true coefficient. Note the inclusion in this figure of a new estimator, "Analytical," which just divides the naive estimates by $1-F N R-F P R$ for both slope coefficients. The analytical estimator offers a good approximation of the true coefficient-much closer to the truth compared to the naive estimates.

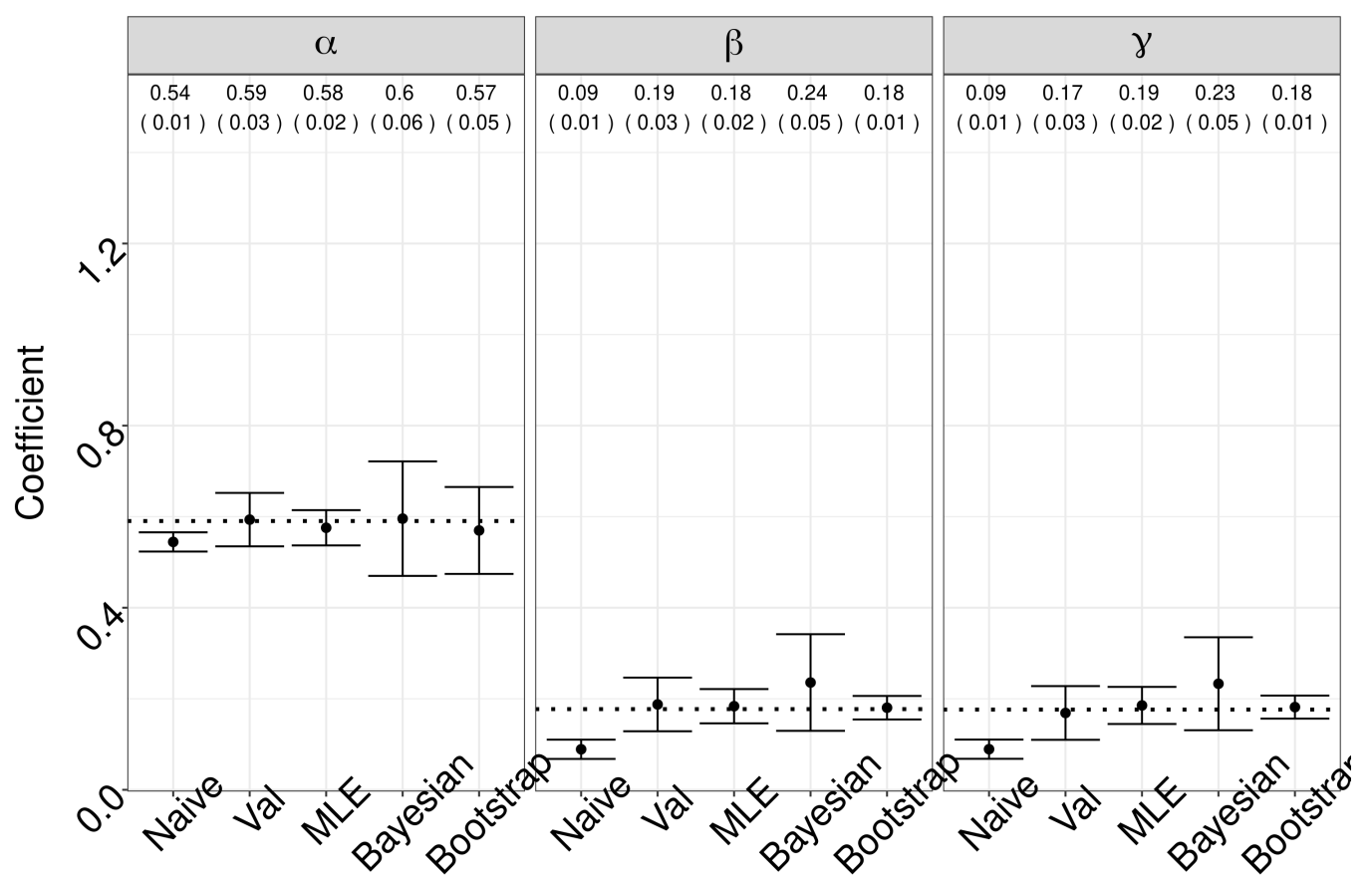

Figure L.1: Estimated regression coefficients with 95\% confidence (credible) intervals when ML classification is used as the outcome in linear regression. The point estimates and their standard errors are listed on the top of each group. The each coefficient is plotted as a dotted horizontal line. 


\section{Appendix M Correlated ML classification and con- trol variables}

In order to generated data that contain correlated confounder and prediction errors, we modified the data generating process from Simulation 1 and 2. The simulations used Cholesky decomposition, which takes a fixed Pearson correlation coefficient and a vector as input, and outputs another vector that has the given correlation coefficient with the input vector.

\section{M.1 Predicted outcome variable}

- $D, W, \epsilon \sim N(0,1)$;

- $Y^{*}=\operatorname{logit}^{-1}(\alpha+\beta D+\gamma W+\epsilon)$, where $\alpha=0.5, \beta=1$, and $\gamma=1$;

- $Y \sim \operatorname{Bernoulli}\left(Y^{*}\right)$

- $e=$ Cholesky $(W, \rho)$, where $r h o$ is ranges from -.75 to 1.25 , with distance 0.25 . In this way, we generated an prediction error $e$ that has the given correlation coefficient with the confounder $W$.

- $Y_{\text {error }}^{*}=e+Y^{*}$. $Y_{\text {error }}^{*}$ is the latent value of predicted outcome variable.

- $\tilde{Y}=\mathbf{I}\left(Y_{\text {error }}^{*}>\operatorname{mean}(Y)\right)$ where $\mathbf{I}$ is an indicator function.

The above DPG will result in the error between latent values of predicted outcome and true outcome $\left(Y_{\text {error }}^{*}-Y^{*}\right)$ and the confounder $W$ has a correlation coefficient exactly equal to $\rho$. Because we have to binarize the latent values, the correlation between prediction error of the binary variable $(\tilde{Y}-Y)$, and confounder $W$, will have a correlation coefficient that is somewhat smaller in scale than $\rho$. 


\section{M.2 Predicted independent variable}

The below data generation process is inspired by Meyer and Mittag (2017, p. 302):

- $D^{*}, \epsilon, \epsilon_{2}, u_{1}, u_{2} \sim N(0,1)$

- $D=\mathbf{I}\left(D^{*}>0\right)$, where $\mathbf{I}$ is an indicator function. $D$ is the true binary independent variable.

- $W=\epsilon+u_{1}$ is a confounder

- $D_{\text {error }}^{*}=D^{*}+\rho \cdot \epsilon+u_{2}$

- $\tilde{D}=\mathbf{I}\left(D_{\text {error }}^{*}>0\right)$ is the predicted binary independent variable.

- $Y=\alpha+\beta D+\gamma W+\epsilon_{2}$, where $\alpha=0.5, \beta=1, \gamma=1$.

Similarly, the above DPG will result in the error between latent values of predicted outcome and true outcome $\left(D_{\text {error }}^{*}-D^{*}\right)$ and the confounder $W$ has a correlation coefficient exactly equal to $\rho$. Because we have to binarize the latent values, the correlation between prediction error of the binary variable $(\tilde{D}-D)$, and confounder $W$, will have a correlation coefficient that is somewhat smaller in scale than $\rho$.

\section{Appendix N More simulations on correlated confounder and prediction error}

This section lists some additional simulations to evaluation how violation of Assumption 1 impacts our estimators. 


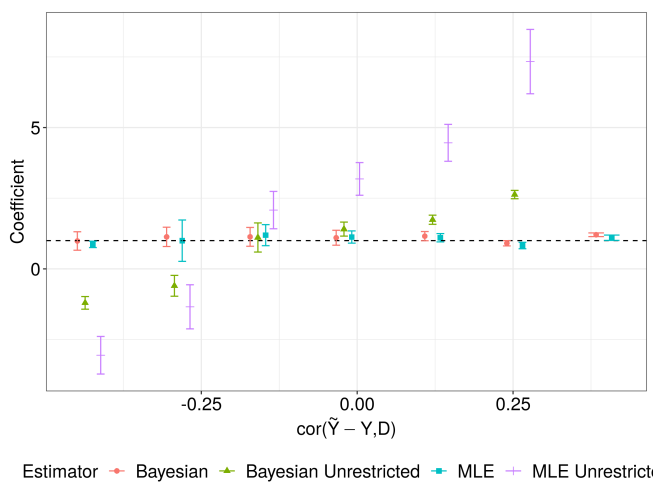

(a) Predicted outcome.

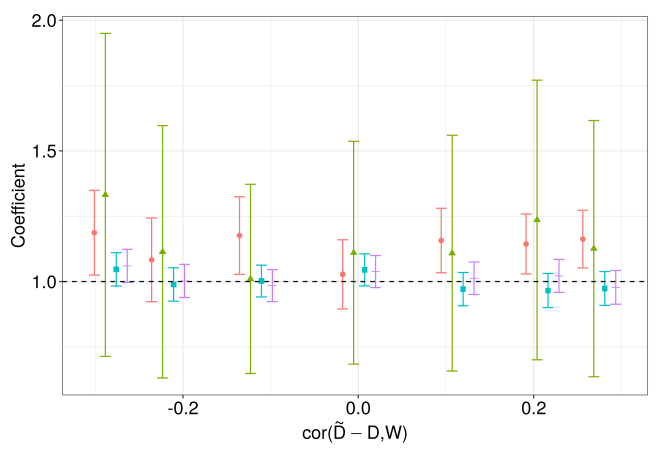

Estimator - Bayesian * Bayesian Unrestricted * MLE + MLE Unrestric

(b) Predicted independent variable.

Figure N.1: Estimated regression coefficients with 95\% confidence (credible) intervals. The true value of each coefficient is plotted as a dotted horizontal line.

\section{N.1 The impact of bounding coefficient estimates}

As we discuss in Section 5.3 to 5.5, it will be useful to restrict the MLE and Bayesian methods to let them find parameter values within the range set by the $95 \%$ confidence interval of the regression using validation data. When Assumption 1 (1A) is satisfied, this requirement actually does not matter; the optimization algorithm can successfully identify the correct point estimates. However, the importance of restricting parameter values within a reasonable range is reflected when Assumption 1(1A) is violated.

Figure N.1 compares the estimated coefficients of MLE and Bayesian estimators, without and with restricting the optimization algorithms to find values within the $95 \%$ confidence interval of the naive regression. It can be seen that if we did not bound MLE and Bayesian estimator, then they will produce biased estimates for predicted outcome case (not for predicted independent variable case). But a simple operation to bound the range of allowed coefficients can make the estimates good again (these are the MLE and Bayesian results we have shown in the main text, Figure 6). 


\section{N.2 Impact of primary data size on coefficients}

One anonymous reviewer's concern is that when an regressor is binary, the outcome has two peaks, and its probability density is a mixture of two densities (i.e. $P(Y \mid D=$ 1) and $P(Y \mid D=0))$. The Bayesian and MLE estimators essentially try to separate the two marginal distributions and use the difference as the estimate as the estimate of. This intuition provides a correct solution if the model is correctly specified, but the reviewer is concerned that if there is misspecification in the function form of $Y$ (e.g. the outcome is not normally distributed), the Bayesian and MLE estimators still seek to find the two peaks, and it is likely to give biased and inconsistent estimates. Furthermore, the reviewer also predicts that "holding the size of the validation sample and the accuracy of the classifier fixed, as the primary sample grows, the performance of the estimator degrades (a kind of anti-consistency)"

In the main article, we have already provided evidence that model misspecification will bias the coefficient estimates, but naive estimates have much larger biases in general. Here, we investigate the second prediction of the reviewer that performance of our proposed estimator degrades with the size of the primary data. Figure N.2 shows the results. We cannot conclude that the increase in the size of the primary data will lead to a higher level of bias. Note that we are not saying that our estimators are not biased; these are known limitations of our estimators because it requires Assumption 1. But there is no evidence that increasing primary data size leads to more biases.

Figure N.3 further increased the sample size to one million. Because Bayesian estimator is too slow, we only simulated how MLE performs with such a large dataset (the Bootstrap estimator relied on MLE hence its point estimates will be similar). Again, we did not find evidence to support the anti-consistency hypothesis: that increasing primary dataset size leads to higher biases of our MLE estimator. 


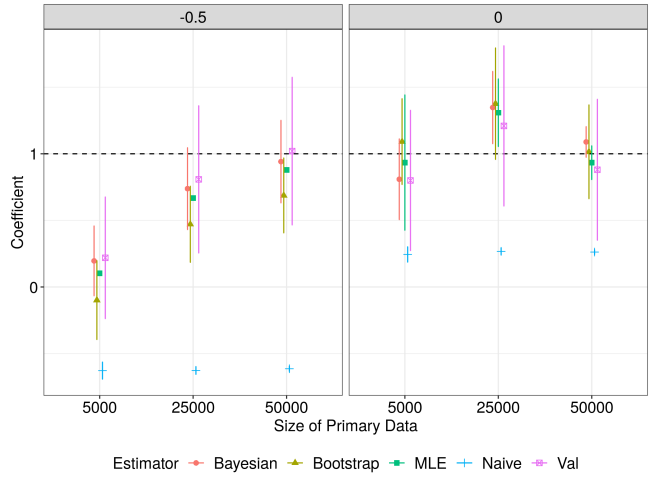

(a) Predicted outcome; observed correlated confounder.

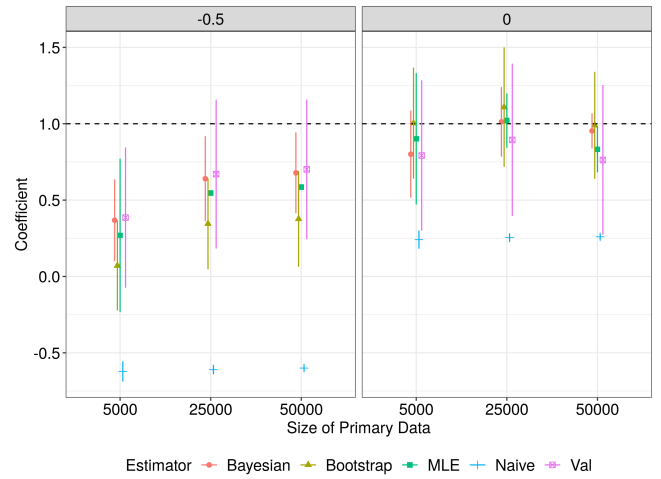

(c) Predicted outcome; unobserved correlated confounder.

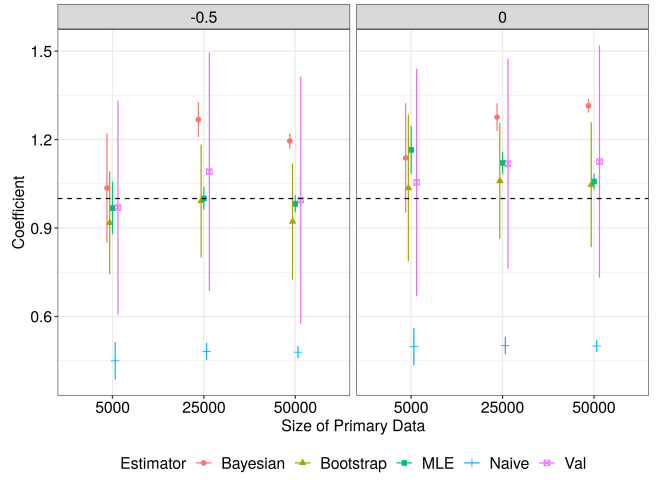

(b) Predicted independent variable; observed correlated confounder.

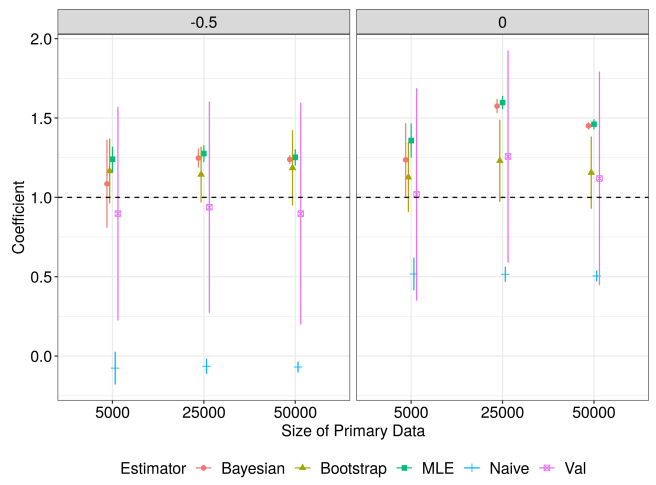

(d) Predicted independent variable; unobserved correlated confounder.

Figure N.2: The effect of size of primary data size (x-axis) on coefficient estimates, when observed or unobserved confounder is correlated or uncorrelated with the ML algorithm prediction error. The top of each panel list the Pearson's correlation coefficient between confounder and prediction errors. Estimated regression coefficients with $95 \%$ confidence (credible) intervals. The true value of each coefficient is plotted as a dotted horizontal line. 


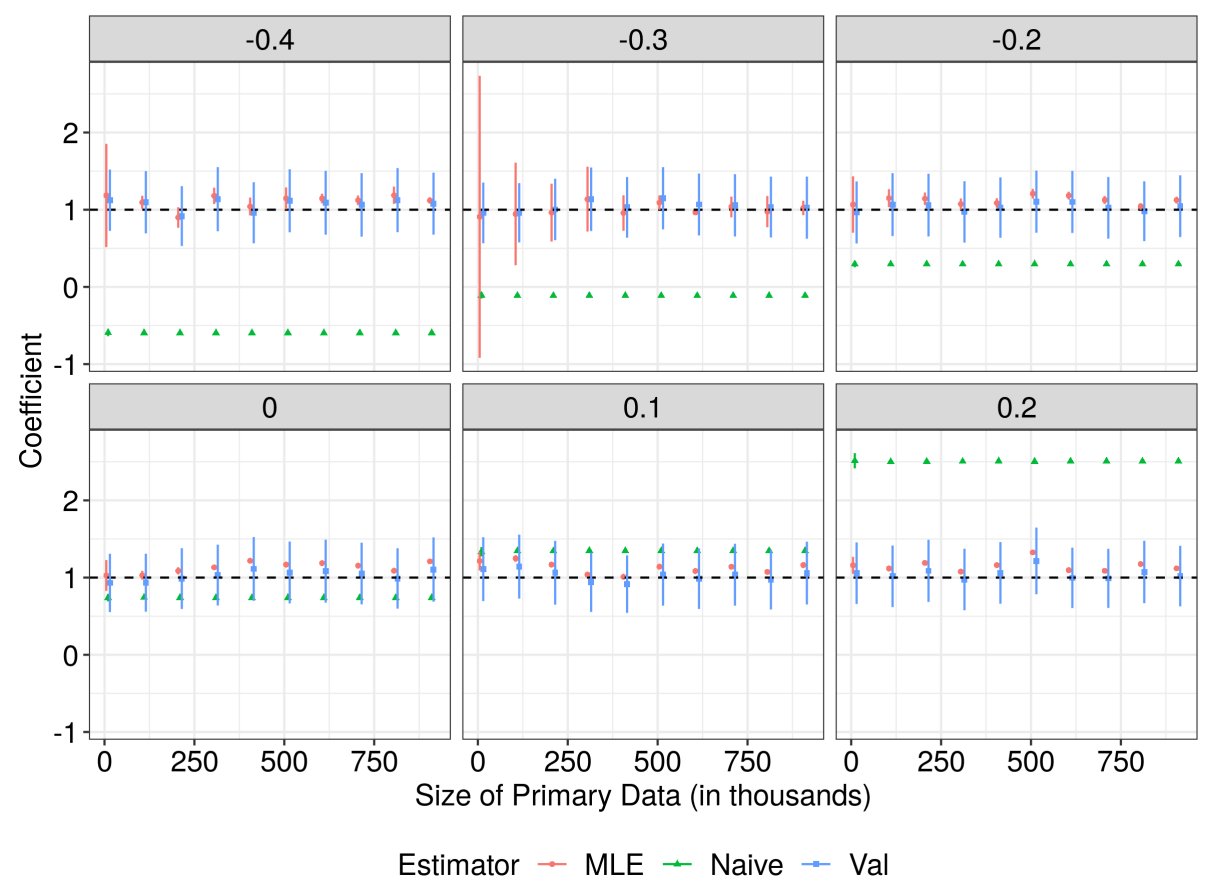

(a) Predicted outcome; observed correlated confounder.

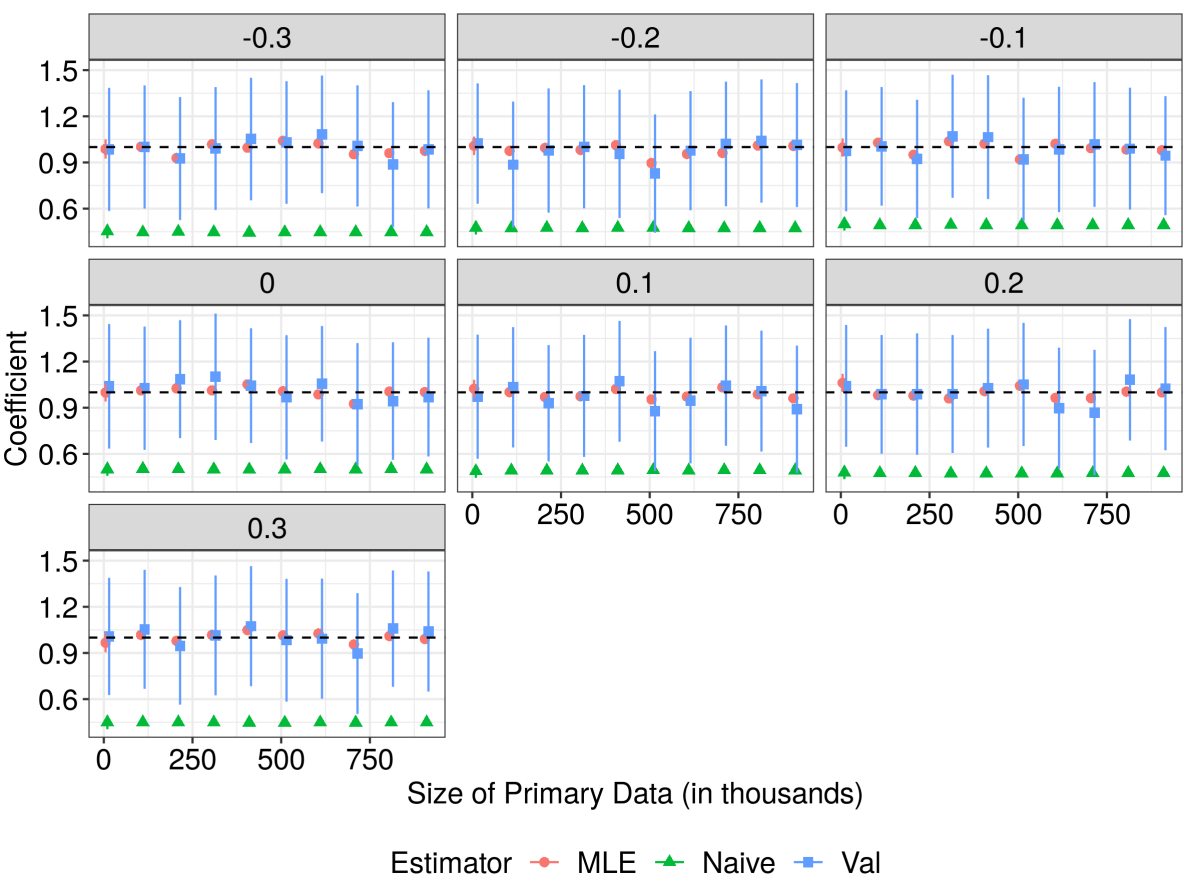

(b) Predicted independent variable; observed correlated confounder.

Figure N.3: The effect of size of primary data size (x-axis) on coefficient estimates, when observed or unobserved confounder is correlated with the ML algorithm prediction error. The top of each panel list the Pearson's correlation coefficient between confounder and prediction errors. Estimated regression coefficients with $95 \%$ confidence (credible) intervals. The true valug ${ }_{4}$ of each coefficient is plotted as a dotted horizontal line. 


\section{Appendix O Reestimation of real-world examples}

\section{O.1 Cantu (2019)}

Cantu provided FNR and FPR of his ML algorithm in his Table 1. It allowed us to use all four estimators we proposed.

The three key independent variables in the regression are:

- "totalexperience": a dummy indicating whether the state executive had previously held an elected public office.

- "noOP": a dummy indicating if no representative from the opposition party had signed the tally.

- "centeno": indicating whether identifies those governors were within Salinas's political group. This information comes from Centeno (2004), who identifies 40 top-level officials in the Salinas's camarilla.

\section{O.2 Fowler et al. (2020)}

Fowler et al. (2020) (Study 2) is interested in tone differences between political advertisements on Facebook and television. They measured tone in three different ways: promoting self, attacking others, or contrasting self with others. They obtained binary classifications for each of the three types of tones for every advertisement, took the average tone across each candidates' advertisement on Facebook and television, and used them as outcome variables.

Fowler et al. (2020, Figure B.1) provided FNR and FPR of their predictions, but it was not easy to read the detailed numbers of the plot. We found the exact values of FNR and FPR for each of the three tone measures from the authors' replication 
files on Dataverse (the file name is called "cv_error_rates_dropout.RData"). Then we used Equation 2 to obtain the mean tone for each candidate. Ideally, we would like to obtain candidate-level error rates, but as we explained in Footnote 17 in the main text, it was not provided in Fowler et al. (2020) and would be very challenging to estimate. Hence, we had to assume that the error rates are the same across candidates.

\section{O.3 Mitts (2019)}

For Mitts (2019), the outcome variable is the number of polarized tweets. There were five measures to determine whether each tweet is polarized or not. We used the first among the five to determine the extent to which the tweet expressed anti-west sentiment. Mitts (2019) provide four sets of $F N R / F P R$ depending on the language of the tweets: English, French, German, or Arabic), listed in her Table S12 - S15. Depending on the language of the tweet, we used the corresponding FNR and FPR and Equation 2 to estimate the proportion of radicalized tweets for each user. Then we time the proportion by the number of total posts to obtain the counts of radicalized tweets for each user.

Because the level of aggregation is at the user level, it would be ideal to obtain user-level error rate estimates. This will allow for a more accurate estimation of the number of polarized tweets from each user. However, it would be unbearably time consuming to calculate such user-level error rates, so Mitts (2019) did not provide that.

The independent variables in Mitts (2019) come from survey and administrative data. They are: percentage of unemployment ("unemployed_pct"), share of far-right votes ("right_wing_pct"), and percentage of foreigners ("foreign_pct"). 


\section{O.4 Katagiri and Min (2019)}

Katagiri and Min (2019) are interested in the relative efficacy of private statements, public statements, and material actions during the Berlin Crisis of 1958-63. One other independent variable (whether there is material action) is obtained from New York Times reports(or "nyt" in Figure 9d) and measured from expert coding (whether the action reflected hostility toward the Soviet Union), so we treat this as error-free.

The dependent variable and two key independent variables were generated by ML classification. The two predicted independent variables are: private statements, obtained from declassified telegrams from the U.S. Department of Defense ("dos"); and public statement during the Berin Crisis, obtained from the Foreign Broadcast Information Service ("fbis"). The dependent variable is the White House's assessment of evaluations of the Soviet Union's resolve, that is, willingness to use force to achieve its aims in Berlin. The authors first classified the private statements, public statements, as well as the White House's assessment into a binary classification: whether they expressed willingness to use force with respect to Berlin. The three variables were then aggregated at the weekly level as the number of documents expressing willingness to use force in the private, public, and White House statements.

Appendix $\mathrm{C}$ of Mitts (2019) provides measures for $F N R$ and $F P R$ of the three predicted independent/dependent variables. We used these $F N R$ and $F P R$ and Equation 2 to first estimate the proportion of documents that expressed willingness to use force. Then we time these proportions with the number of total documents in private, public, and White House documents to obtain the weekly counts. These corrected weekly counts were used in the regression. Finally, we note that the authors used negative binomial regression and we followed them.

Still, ideally, we would want to obtain the error rates by week since that is the 
level of aggregation. However, Mitts (2019) does not provide these numbers, and it would not be easy to calculate. Therefore, we have to assume that the error rates were the same across each week. This assumption might be violated, but until there was a better algorithm to estimate group-specific error rates, we have to proceed with this assumption.

In the main text, we found that none of the three independent variables had statistically significant effects on the outcome: the White House' willingness to use force. We replicated another model of the author (Model 2 in Table), which used the same data but leveraged the Poisson autoregressive model to better model the time series data. Figure O.1 shows that using corrected count measures and this more complex autoregressive model greatly widens the confidence interval estimates, again making none of the results statistically significant. 


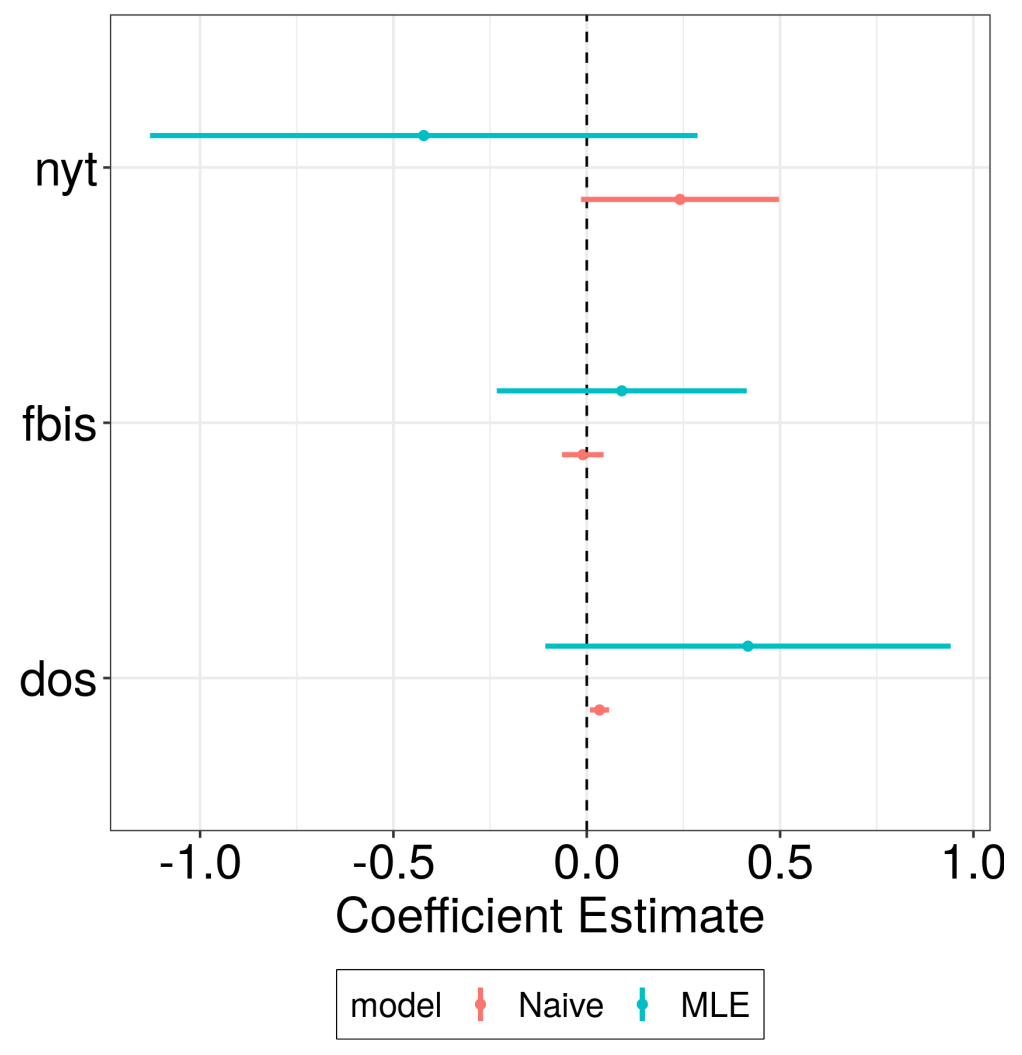

Figure O.1: Katagiri and Min (2020), Model 2 in Table 3. 


\section{References for Appendix}

Bandiera, Oriana, Andrea Prat, Stephen Hansen, and Raffaella Sadun. 2020. "CEO Behavior and Firm Performance." Journal of Political Economy 128:1325-1369.

Barberá, Pablo, Andreu Casas, Jonathan Nagler, Patrick J. Egan, Richard Bonneau, John T. Jost, and Joshua A. Tucker. 2019. "Who Leads? Who Follows? Measuring Issue Attention and Agenda Setting by Legislators and the Mass Public Using Social Media Data." American Political Science Review 113:883-901.

Fligstein, Neil, Jonah Stuart Brundage, and Michael Schultz. 2017. "Seeing Like the Fed: Culture, Cognition, and Framing in the Failure to Anticipate the Financial Crisis of 2008." American Sociological Review 82:879-909.

Flores, René D. 2017. "Do Anti-Immigrant Laws Shape Public Sentiment? A Study of Arizona's SB 1070 Using Twitter Data." American Journal of Sociology 123:333384.

Karell, Daniel and Michael Freedman. 2019. "Rhetorics of Radicalism." American Sociological Review 84:726-753.

Pan, Jennifer and Kaiping Chen. 2018. "Concealing Corruption: How Chinese Officials Distort Upward Reporting of Online Grievances." American Political Science Review 112:602-620. 